\title{
A Panchromatic View of the Bulge Globular Cluster NGC 6569*
}

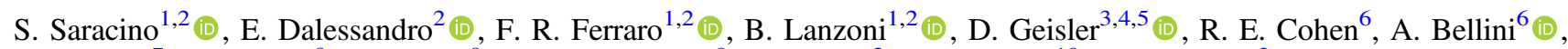 \\ E. Vesperini ${ }^{7}$, M. Salaris ${ }^{8}$, S. Cassisi ${ }^{9}$ (D), A. Pietrinferni ${ }^{9}$, L. Origlia ${ }^{2}$ (D), F. Mauro ${ }^{10}$, S. Villanova ${ }^{3}$ (D), and C. Moni Bidin ${ }^{10}$

\begin{abstract}
We used high-resolution optical Hubble Space Telescope/WFC3 and multiconjugate adaptive optics assisted GEMINI GeMS/Gemini South Adaptive Optics Imager observations in the near-infrared (NIR) to investigate the physical properties of the globular cluster (GC) NGC 6569 in the Galactic bulge. We have obtained the deepest purely NIR color-magnitude diagram published so far for this cluster using ground-based observations, reaching $K_{s} \approx 21.0$ mag (two magnitudes below the main-sequence turn-off point). By combining the two data sets secured at two different epochs, we determined relative proper motions for a large sample of individual stars in the center of NGC 6569, allowing a robust selection of cluster member stars. Our proper motion analysis solidly demonstrates that, despite its relatively high metal content, NGC 6569 hosts some blue horizontal branch stars. A differential reddening map has been derived in the direction of the system, revealing a maximum color excess variation of about $\delta E(B-V) \sim 0.12 \mathrm{mag}$ in the available field of view. The absolute age of NGC 6569 has been determined for the first time. In agreement with the other few bulge GCs with available age estimates, NGC 6569 turns out to be old, with an age of about $12.8 \mathrm{Gyr}$, and a typical uncertainty of $0.8-1.0 \mathrm{Gyr}$.
\end{abstract}

Key words: globular clusters: individual (NGC 6569) - instrumentation: adaptive optics - proper motions techniques: photometric

\section{Introduction}

The Galactic bulge is one of the most inaccessible regions of the Milky Way and its structure, formation, and evolution are still subjects of intense debate within the astronomical community (see, for example, Rich 1998; Ness et al. 2013; Origlia 2014; Zoccali \& Valenti 2016). Many studies have shown that globular clusters (GCs) orbiting the bulge are key tools to trace the properties of the bulge stellar population in terms of kinematics, chemical abundances, and age (Bica et al. 2006; Valenti et al. 2007; Barbuy et al. 2018). Moreover, metal-rich bulge GCs represent the ideal local templates for studying the stellar content of extra-Galactic unresolved bulges and elliptical galaxies. Unfortunately, because of observational limitations mainly related to the large extinction and stellar density in the direction of the bulge, its GCs have been systematically excluded from large surveys and remain poorly investigated. The advent of high-resolution near-infrared (NIR) imagers equipped with Multi Conjugate Adaptive Optics (MCAO) facilities mounted at ground-based $8-10 \mathrm{~m}$ class

\footnotetext{
Based on observations obtained at the Gemini Observatory, which is operated by the Association of Universities for Research in Astronomy, Inc., under a cooperative agreement with the NSF on behalf of the Gemini partnership: the National Science Foundation (United States), the National Research Council (Canada), CONICYT (Chile), the Australian Research Council (Australia), Ministério da Ciência, Tecnologia e Inovação (Brazil) and Ministerio de Ciencia, Tecnología e Innovación Productiva (Argentina). Based on observations (GO 15232, PI: Ferraro) with the NASA/ESA Hubble Space Telescope, obtained at the Space Telescope Science Institute, which is operated by AURA, Inc., under NASA contract 5-26555.
}

telescopes opened a new line of investigation, arousing the interest of astronomers in the Galactic bulge and its bulge GC system (e.g., Ferraro et al. 2009b; Saracino et al. 2015, 2016, see also a complementary study based on deep NIR observations with the Hubble Space Telescope (HST) recently carried out by Cohen et al. 2018).

Moreover, ground-based diffraction-limited observations provide very high spatial resolution (higher than $H S T$, due to the larger apertures of the $8 \mathrm{~m}$ class telescopes) and thus they can be used for accurate proper motion (PM) studies. However, while the exceptional astrometric performance of $H S T$ has been extensively exploited during the last $20 \mathrm{yr}$, allowing us to investigate the kinematics and dynamics of a large sample of stellar systems (see, e.g., McLaughlin et al. 2006; Anderson \& van der Marel 2010; McNamara et al. 2012; Bellini et al. 2014; Watkins et al. 2015; Libralato et al. 2018), only a few PM studies using MCAO observations exist in the literature (Ortolani et al. 2011; Massari et al. 2016a; Fritz et al. 2017; Monty et al. 2018).

The work presented here is focused on the GC NGC 6569 and it is part of an extended project aimed at characterizing the stellar populations of a sample of highly extincted stellar systems in the Galactic bulge (see the extensive work done in Terzan 5; Ferraro et al. 2009b, 2015, 2016; Lanzoni et al. 2010; Origlia et al. 2011, 2013; Massari et al. 2012, 2014a, 2014b) orbiting the innermost regions of our Galaxy.

NGC 6569 is a moderately compact globular (with a concentration parameter $c=1.27$, Trager et al. 1995) located 


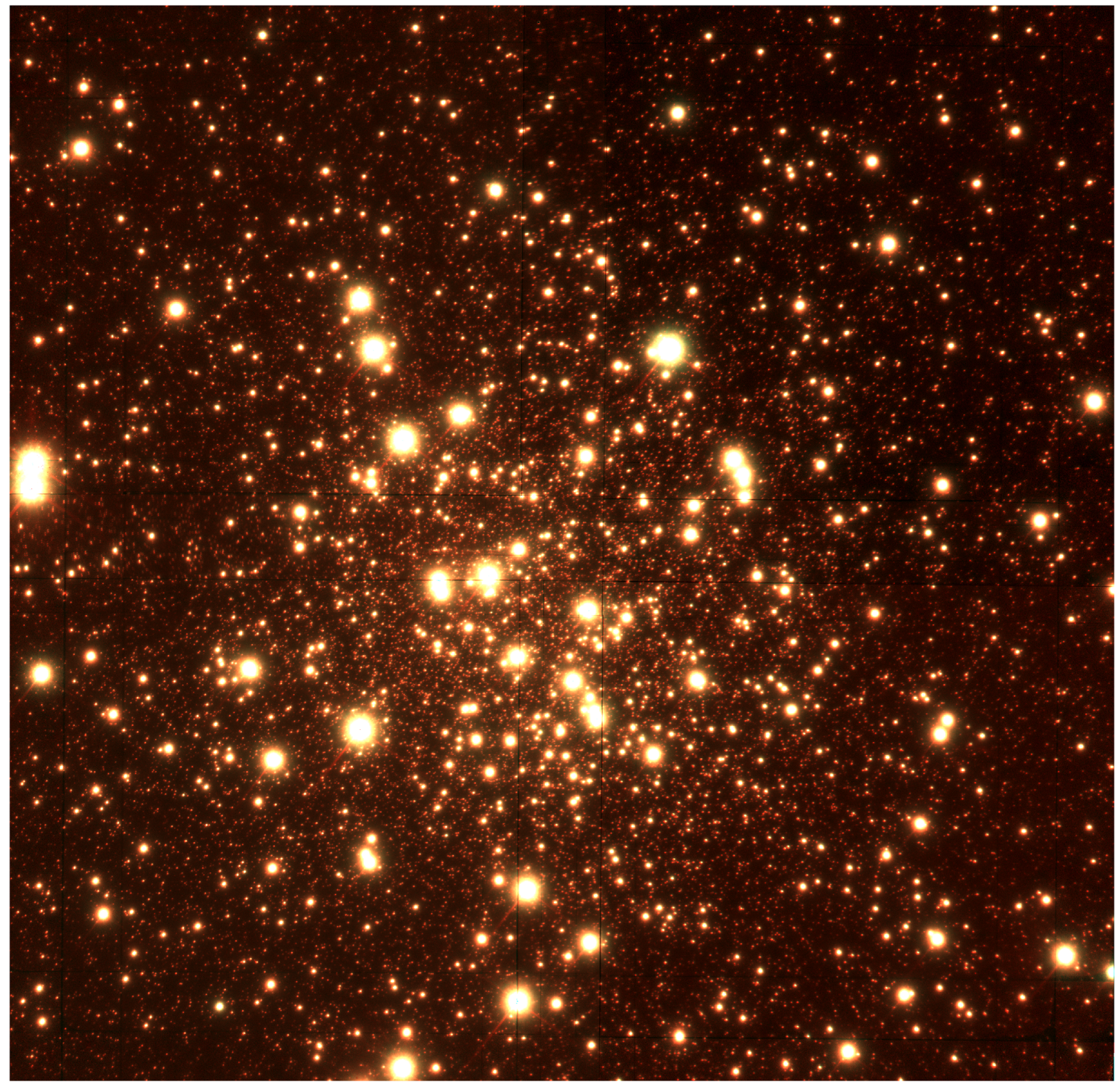

Figure 1. False-color image of NGC 6569 obtained by combining GEMINI observations in the near-IR $J$ and $K_{s}$ bands. North is up; east is to the left. The FOV is $92^{\prime \prime} \times 92^{\prime \prime}$.

in the Sagittarius region $\left(l=0.48, b=-6^{\circ} .68\right.$; Harris 1996, 2010 edition), at a distance of only $3 \mathrm{kpc}$ from the Galactic center (Harris 1996). The cluster is projected toward the dark nebula Barnard 305 (Barnard et al. 1927) and it is therefore highly reddened, with an average color excess $E(B-V)=0.53$ (Ortolani et al. 2001). The first optical (V, V-I) color-magnitude diagram (CMD) of NGC 6569 was obtained (Ortolani et al. 2001) by using data from the $1.5 \mathrm{~m}$ Danish telescope at ESO La Silla. These data sample only the brightest portion of the CMD down to the red horizontal branch (HB). Deeper CMDs have been obtained in the optical by Piotto et al. (2002) and in the NIR by Valenti et al. (2005) using the HST and the New Technology Telescope at ESO La Silla, respectively. However, these observations are unable to properly characterize the mainsequence turn-off (MS-TO) region of the cluster. A more recent photometric analysis of the cluster was performed by Mauro et al. (2012, see also Cohen et al. 2017) using $J, H$, and $K_{s}$ data from the Vista Variables in the Via Lactea (VVV) survey. Interestingly, the authors found evidence of the presence of two red HBs separated by $\approx 0.1 \mathrm{mag}$ in the $K_{s}$ band. In a very recent work performed using the IR channel of the HST/WFC3, Cohen et al. (2018) imaged the MS of NGC 6569 also in the NIR, sampling a few magnitudes below the MS-TO and highlighting the presence of an extension of the HB blueward of the red clump.

From low-resolution spectroscopy, Zinn \& West (1984) measured a metallicity $[\mathrm{Fe} / \mathrm{H}]=-0.8$ for the cluster. A similar value $([\mathrm{Fe} / \mathrm{H}]=-0.79 \pm 0.02)$ was obtained later from highresolution IR spectra of six stars by Valenti et al. (2011), with an average $\alpha$-element enhancement of $+0.43 \pm 0.02$. The most 


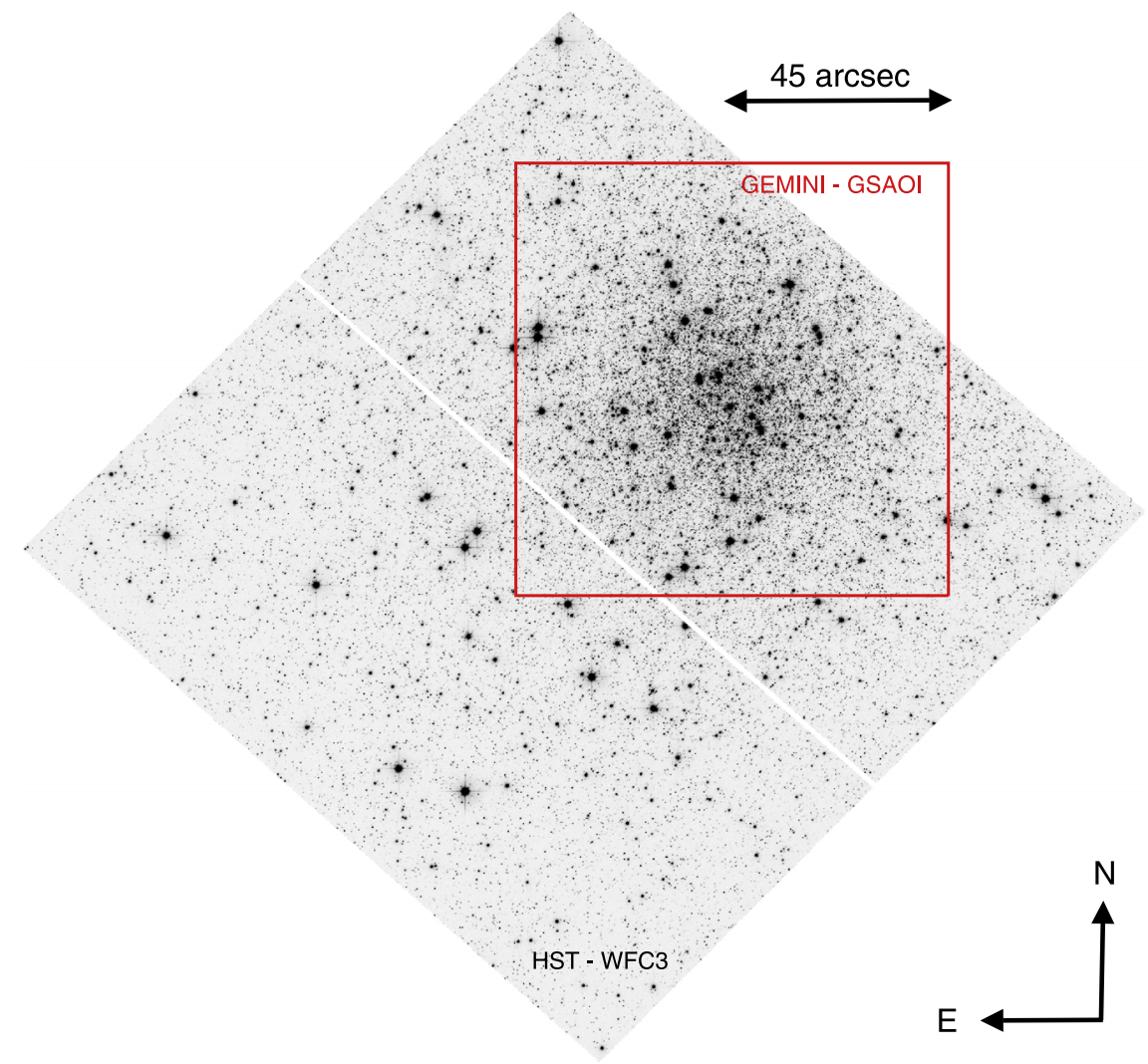

Figure 2. HST/WFC3 image of NGC 6569 in the F814W filter. North is up, east is to the left. The WFC3 FOV is $162^{\prime \prime} \times 162^{\prime \prime}$. The red box corresponds to the GSAOI/GEMINI FOV, of $92^{\prime \prime} \times 92^{\prime \prime}$ on the sky.

recent determination has been published by Johnson et al. (2018), who studied a sample of 19 stars with the Magellan-M2FS and the VLT-FLAMES spectrographs and found a mean $[\mathrm{Fe} / \mathrm{H}]=$ -0.87 , with $[\alpha / \mathrm{Fe}] \sim+0.4$.

One of the most surprising characteristics of NGC 6569, given its relatively high metallicity, is the presence of a quite populated family of RR Lyrae stars. The first evidence dates back to Rosino (1962), who identified eight variable stars, of which six lie within the tidal radius of the cluster. Later on, Hazen-Liller (1984, 1985) and Kunder et al. (2015) discovered a sizeable population of 27 RR Lyrae variables within 8 arcmin from the cluster center. The proposed explanation for the large population of RR Lyrae was a possibly older age of NGC 6569 with respect to other GCs of the same metallicity (Zinn 1980). However, the age of the cluster has never been estimated.

In this work we present the first determinations of (1) relative PMs for a large sample of individual stars in the cluster core based on HST and GEMINI observations, allowing a solid separation between cluster members and field contaminants, (2) the differential reddening map in the direction of the system, and (3) the absolute age of the cluster. In Section 2 we describe the NIR and optical observations used in this work and the adopted data analysis. Section 3 presents the derived optical-NIR CMDs of NGC 6569, with a discussion of its main stellar populations. The PM analysis is described in Section 4. Section 5 is focused on the determination of the differential extinction in the direction of the cluster. In Section 6 we derive the distance modulus of NGC 6569 by means of two independent methods. The age of the cluster is determined in Section 7 , by applying the MS-fitting method to different sets of theoretical models. In Section 8 we present our summary and conclusions.

\section{Observations and Data Analysis}

\subsection{NIR and Optical Data Sets}

The NIR photometric data used in the present study consist of a set of high-resolution images obtained in 2013 May with the Gemini South Adaptive Optics Imager (GSAOI) assisted by GeMS at the $8 \mathrm{~m}$ Gemini South Telescope, as part of the GEMINI program GS-2013-Q-23, PI: D. Geisler. The camera GSAOI is characterized by four chips and it covers a total FOV of $85^{\prime \prime} \times 85^{\prime \prime}$ on the sky, with a high angular resolution $(0$ ". $02 /$ pixel, Neichel et al. 2014). GeMS uses a constellation of five laser guide stars plus three natural guide stars, to compensate the distortions due to the turbulence of the Earth's atmosphere. We sampled the central region of NGC 6569 with a mosaic of multiple exposures, 14 in both $J$ and $K_{s}$, with exposure time $t_{\text {exp }}=30 \mathrm{~s}$ each. A dither pattern of a few arcseconds has been applied in both filters to recover the gaps among the chips. In Figure 1 we show a two-color image of NGC 6569, obtained by combining GEMINI $J$ - and $K_{s}$-band observations.

The optical data set has been obtained from the UVIS channel of the WFC3, on board HST. The WFC3 UVIS images are characterized by a spatial resolution of 0 ". 04 / pixels and two twin chips covering a total FOV of $162^{\prime \prime} \times 162^{\prime \prime}$ on the sky. The observations have been acquired in 2017, as part of the $H S T$ proposal GO 15232, PI: F.R. Ferraro. They consist of six images in $F 555 \mathrm{~W}$ with $t_{\mathrm{exp}}=30 \mathrm{~s}$ and 6 images in $F 814 \mathrm{~W}$ with $t_{\exp }=13 \mathrm{~s}$. Figure 2 shows a $H S T / \mathrm{WFC} 3$ image in the $F 814 \mathrm{~W}$ 


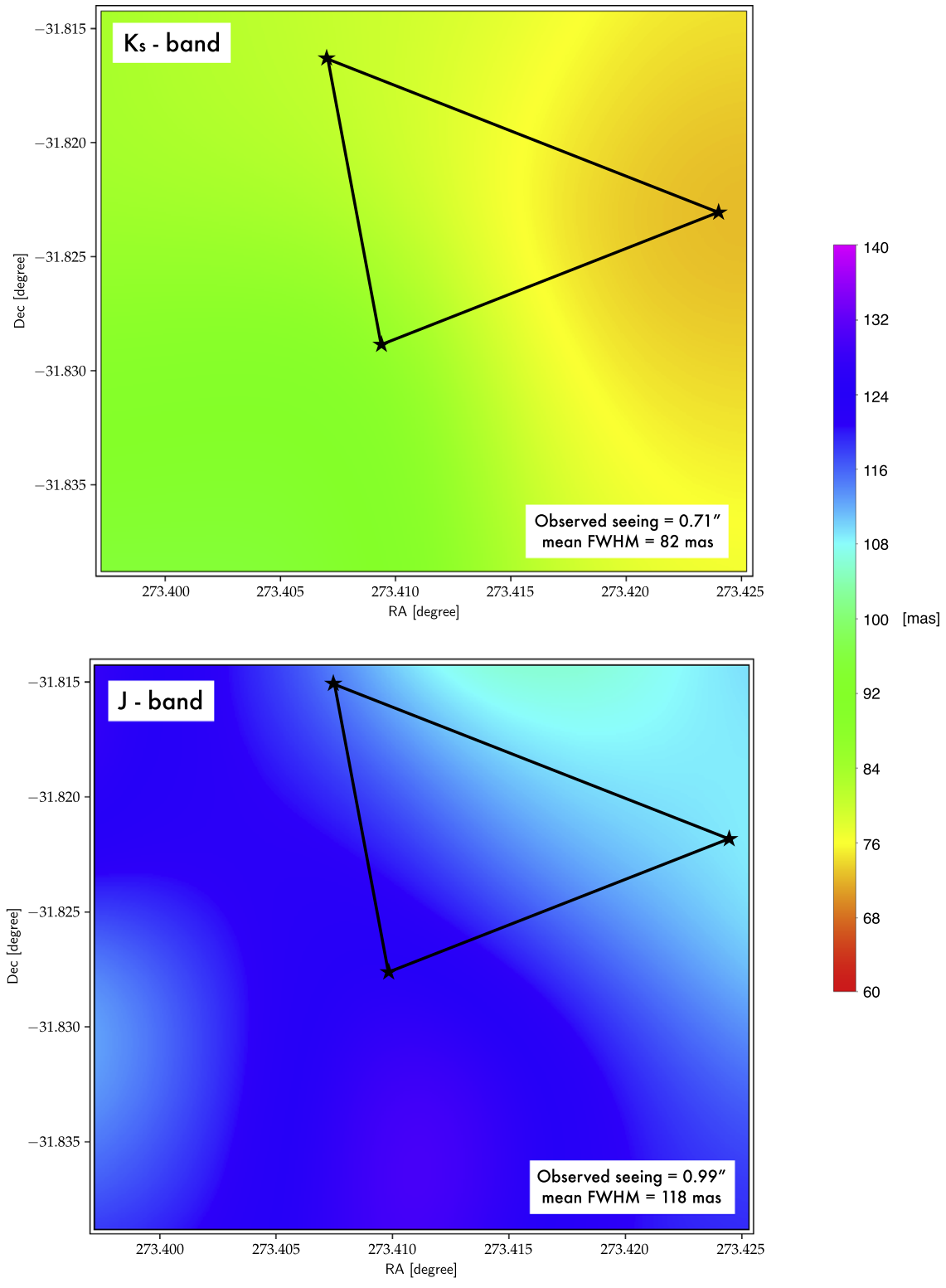

Figure 3. FWHM maps of the best $K_{s}$ image (top panel) and the worst $J$ image (bottom panel). They are based on a sample of $\sim 450$ and 300 stars, in the corresponding mosaic, coadded images. Average FWHM values of 82 mas and 118 mas have been derived for the best and the worst maps, respectively. The tip-tilt NGS stars are presented by black star symbols and connected through solid lines. The observed seeing, after correction for airmass, is marked in the maps. The color code, ranging from magenta (worst) to red (best), is a performance indicator. As can be seen, the best correction is achieved closer to the NGS asterism.

filter, with superimposed the FOV covered by the GEMINI observations discussed above.

\subsection{Data Reduction}

For the NIR data set, we used standard IRAF ${ }^{11}$ (Tody 1986, 1993) tools to correct the raw images for flatfield and to perform the sky subtraction. A master sky was obtained by combining five sky images of a relatively empty field. For the optical data set, instead, we used images processed, flat-fielded, bias subtracted, and corrected for Charge Transfer Efficiency losses by standard HST pipelines (_flc images). The WFC3 images have also been corrected by

\footnotetext{
11 IRAF is distributed by the National Optical Astronomy Observatory, which is operated by the Association of Universities for Research in Astronomy, Inc., under cooperative agreement with the National Science Foundation.
}

the Pixel Area Map using the files available on the HST website. For both NIR and optical data, the photometric reduction was carried out via point-spread function (PSF) fitting techniques in each chip of each image independently, by using DAOPHOTIV (Stetson 1987). The PSF has been modeled by selecting about 200 bright and isolated stars uniformly distributed in each chip, and by using the DAOPHOTIV/PSF routine. We allowed the PSF to vary within each chip following a cubic polynomial spatial variation. The best-fit PSF analytic models obtained for the HST $F 555 W$ and $F 814 W$ images, are a Moffat function with $\beta=1.5$ (Moffat 1969) and a Penny function (Penny 1976), respectively. The typical PSF model for the GEMINI images is a Penny function, but in some cases it was necessary to adopt a Lorentz function (Melcher \& Gerth $1977)$ as well. The PSF models thus obtained were then applied to all the star-like sources detected at a $3 \sigma$ level above the local background by using ALLSTAR. We have derived in this way 

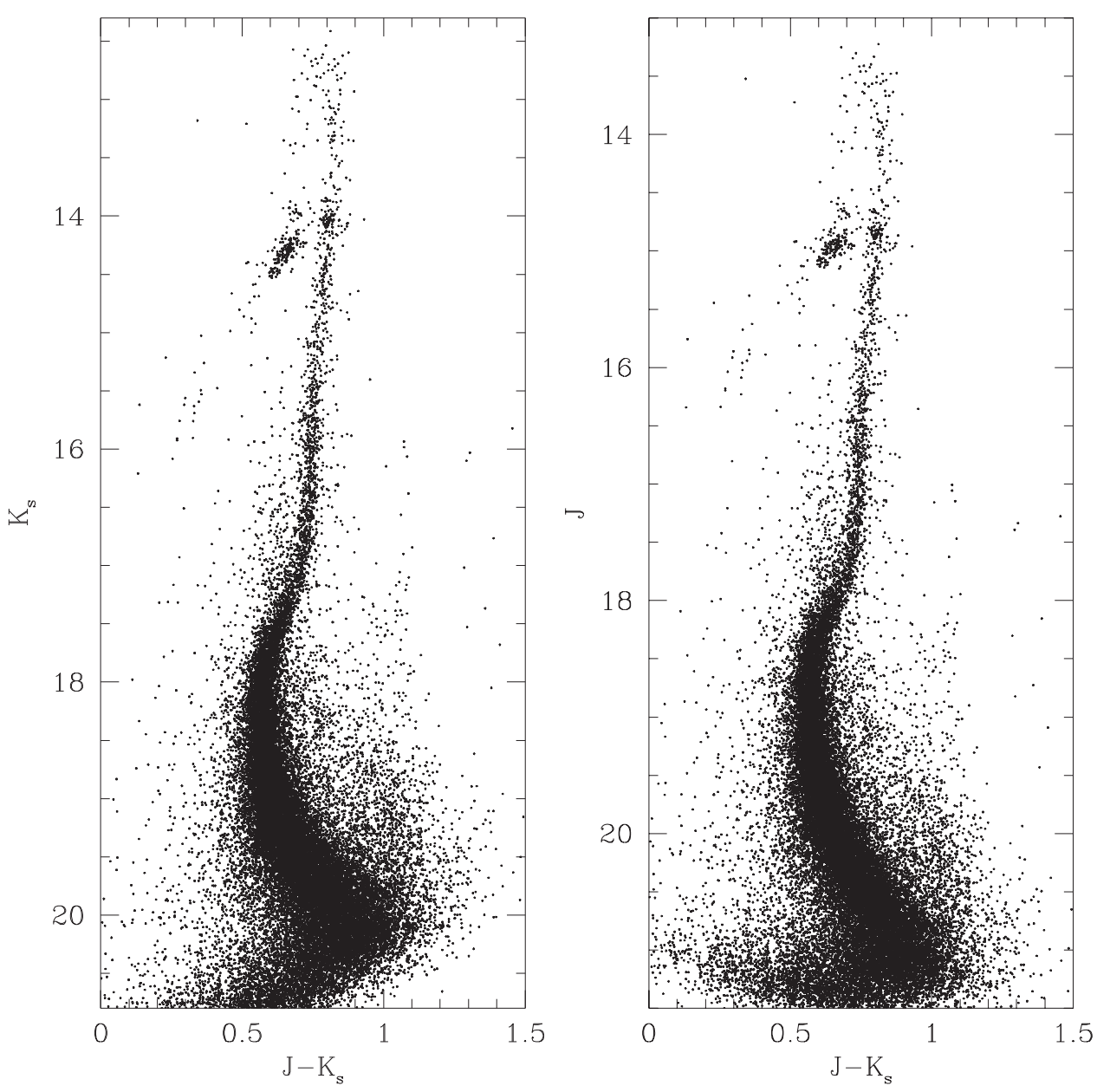

Figure 4. $\left(K_{s}, J-K_{s}\right)$ and $\left(J, J-K_{s}\right)$ CMDs of NGC 6569 obtained from the GEMINI observations discussed in Section 2. All the main evolutionary sequences of the cluster are clearly visible, from the RGB, HB, down to the MS. The quality and the deepness of these NIR CMDs is comparable to those achieved with HST/ WFC3 observations (see Figure 12 in Cohen et al. 2018).

the stellar instrumental magnitudes. Then, starting from the star lists thus obtained and to fill the gaps among the GSAOI chips, we created a GEMINI master star list containing all the stars measured in at least three $K_{s}$ images. For HST instead, the master list was made up of all the stars measured in at least four $F 814 W$ observations. As done in previous works (e.g., Dalessandro et al. 2014 and references therein), the master lists thus created for the two data sets have been used as input for ALLFRAME (Stetson 1994). The files obtained as output have been independently combined to get a GEMINI catalog containing all the stars measured in at least three $J$ and three $K_{s}$ images and an HST catalog containing stars measured in at least four $F 555 W$ and four $F 814 W$ exposures. For every stellar source in each catalog, different magnitude estimates have been homogenized and their mean values and standard deviations have been adopted as the star magnitudes and photometric errors in the final catalog (Ferraro et al. 1991, 1992).

\subsection{Calibration and Astrometry}

The $J$ and $K_{s}$ instrumental magnitudes have been converted into the 2MASS photometric system by using the stars in common with the VVV catalog of NGC 6569 (Mauro et al. 2012; Cohen et al. 2017) as secondary photometric calibrators. The $F 555 W$ and $F 814 W$ instrumental magnitudes have been instead reported to the VEGAMAG photometric system by adopting the zero points listed in the WFC3 website ${ }^{12}$ for a 0 ". 4 (10 pixels) aperture correction. Both catalogs have been finally roto-translated to the absolute (R.A., decl.) coordinates using the stars in common with the VVV catalog ${ }^{13}$ and the crosscorrelation software CataXcorr.

\subsection{GEMINI Performances}

By following the approach used in Dalessandro et al. (2016), we have analyzed the quality of the GEMINI data of NGC 6569. The average Full Width at Half Maximum (FWHM) varies from 3.5 to 5.0 pixels ( 70 mas-100 mas) for the images acquired in the $K_{s}$ band and from 4.0 to 7.0 pixels (80 mas-140 mas) in $J$. For reference, the diffraction-limited FWHMs are 68 mas and 37 mas at $2.2 \mu \mathrm{m}\left(K_{s}\right.$ band) and $1.2 \mu \mathrm{m}$ ( $J$ band), respectively. We have verified that the FWHM is quite stable across the FOV with a maximum variation of $10 \%-15 \%$ in $K_{s}$ and up to $20 \%$ in $J$, in good agreement with what is found in previous works using GEMINI (Saracino et al. 2015, 2016; Massari et al. 2016a). We also verified that the quality of our images strongly

\footnotetext{
12 http://www.stsci.edu/hst/wfc3/phot_zp_lbn

13 The transformation into the absolute reference frame has been doublechecked using the stars in common with the Gaia Data Release 2 catalog.
} 


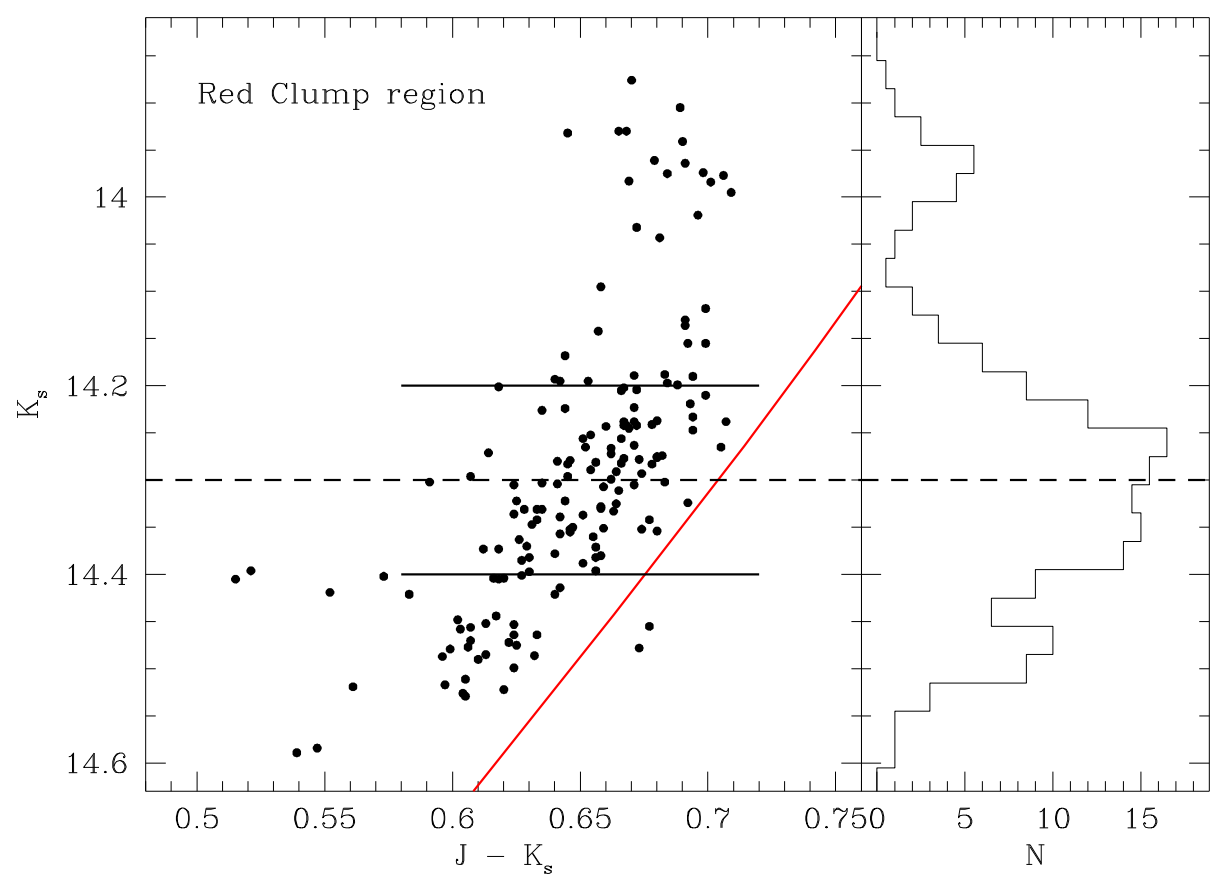

Figure 5. Left panel: $\left(K_{s}, J-K_{s}\right)$ CMD zoomed in the red clump region. The two solid lines mark the brightest and the faintest boundaries of the HB selection boxes used by Mauro et al. (2012). The dashed line marks the magnitude level where the red clump shows the gap in the Hess diagram of Mauro et al. (2012, see their Figure 3). A Zero Age Horizontal Branch (Pietrinferni et al. 2004) for the chemical composition of the cluster is shown in the plot as a red line just to guide the eye through the HB shape in this filter combination. Right panel: Magnitude histogram of the stars observed in the red clump region.

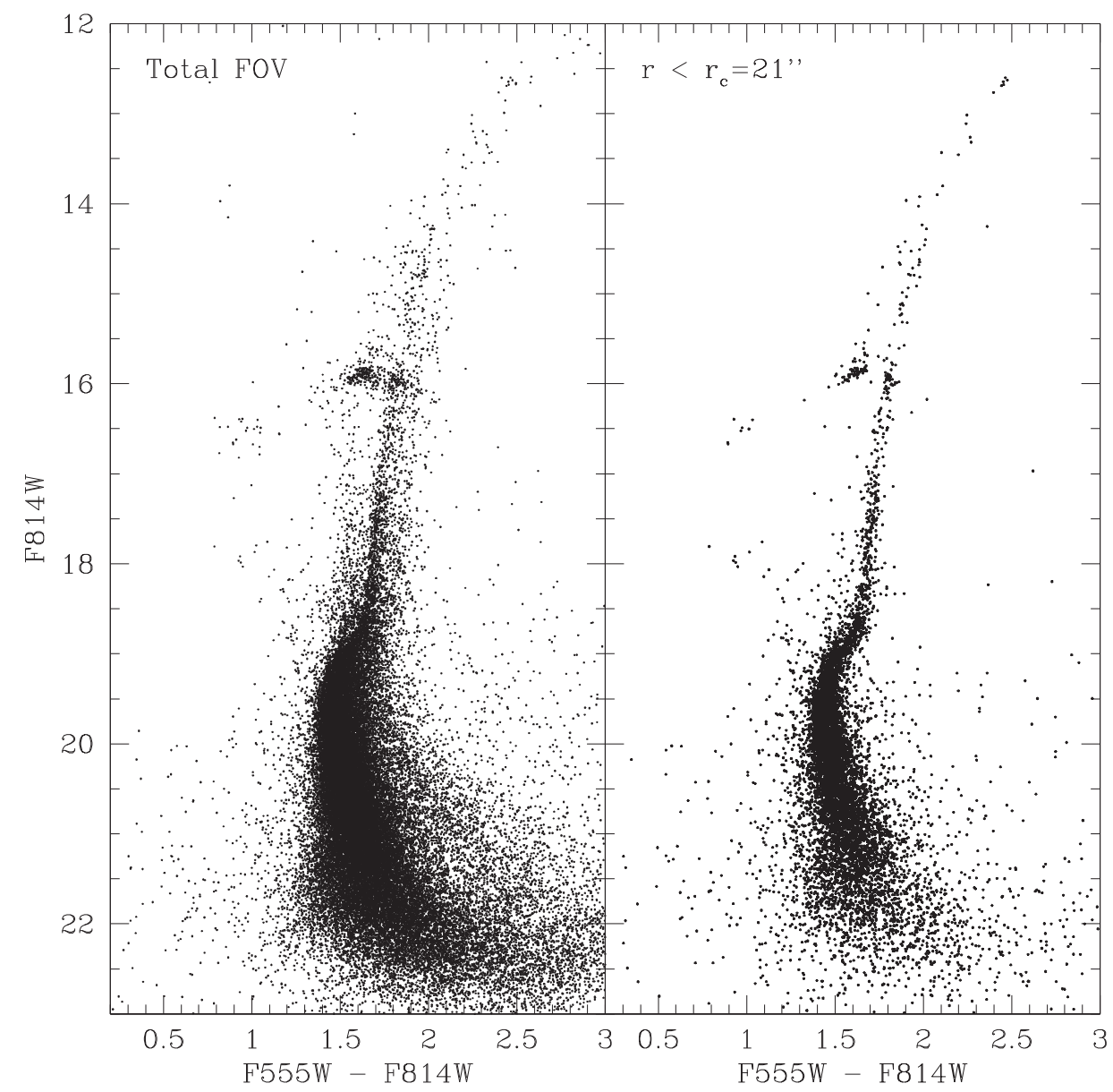

Figure 6. $H S T(F 814 W, F 555 W-F 814 W)$ CMD of NGC 6569 is shown for the total FOV (left panel) and for $r<r_{c}$ (right panel), in order to amplify the differences observed in the central region of the cluster, compared to a more external one. As can be seen, the optical CMD extends for more than 10 mag but field contamination starts to be important going outward. 


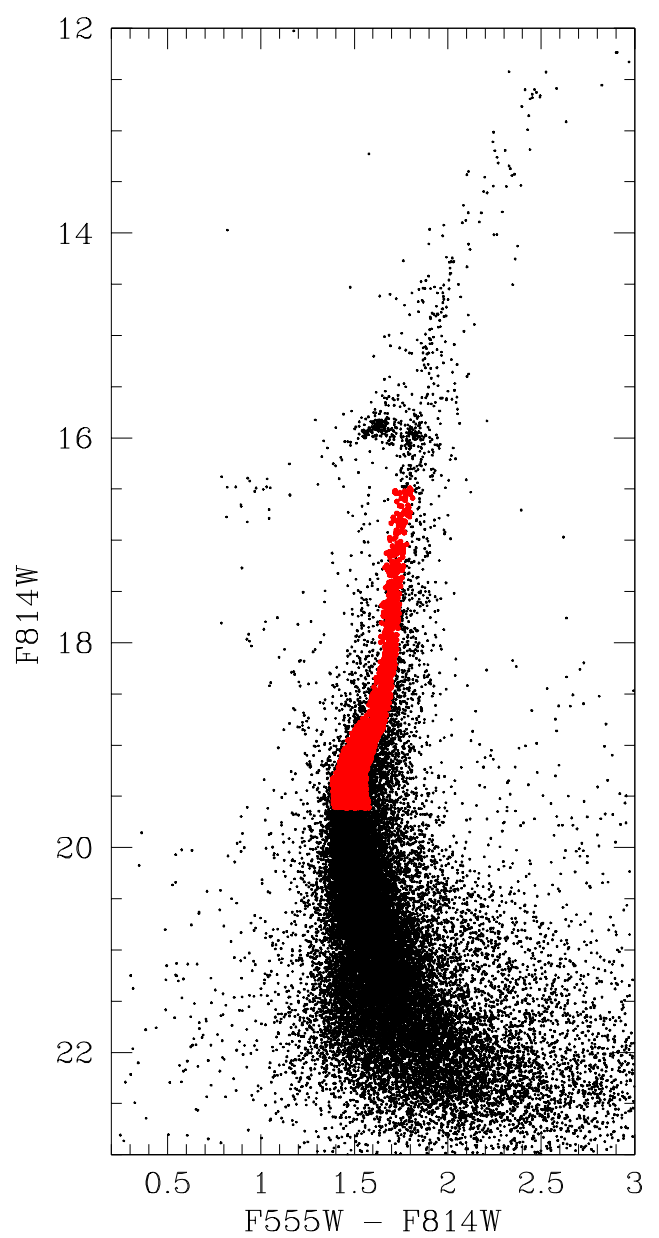

Figure 7. Stars used to create the HST master frame are shown in red on top of the optical $(F 814 W, F 555 W-$ F814W $)$ CMD of NGC 6569. They are likely cluster members on the basis of their position along the lower RGB, the SGB, and the upper MS.

depends on the seeing at the sky position of the target (i.e., at the observed airmass), as already shown in Figure 2 of Dalessandro et al. (2016). We derived the observed seeing as $s(500 \mathrm{~nm}, z)=10.31 /\left(r_{0}(500 \mathrm{~nm}) \times \sec (z)^{-3 / 5}\right)$, where $r_{0}$ is the Fried parameter and $z$ is the observed airmass. In Figure 3 the best $K_{s}$ and the worst $J$ images (in terms of delivered FWHMs) are presented as an example, with the reference guide stars selected for the tip-tilt correction marked as black star symbols.

\section{NIR and Optical CMDs of NGC 6569}

The $\left(K_{s}, J-K_{s}\right)$ and $\left(J, J-K_{s}\right)$ CMDs of NGC 6569 are presented in Figure 4. The comparison with Figure 12 of Cohen et al. (2018) clearly demonstrates the extraordinary capabilities of the GeMS/GSAOI system, that allowed us to obtain NIR CMDs with quality and deepness comparable to those achieved by $H S T /$ WFC3 observations. It is worth noting that the $J$ band is limiting the overall CMD depth. Nevertheless, the CMD spans $\approx 8$ mag, ranging from bright red giant branch (RGB) stars down to low-mass MS stars. Unfortunately, the stars lying along the brightest portion of the $\operatorname{RGB}\left(K_{s}<13.5\right)$ are saturated in all the available images. Instead, the $\mathrm{HB}$, the SGB and the MS-TO $\left(K_{s} \approx 18.0\right)$ are well defined. Unfortunately, our data do not go deep enough to unambiguously identify the MS-Knee (Saracino et al. 2018).
The $\left(K_{s}, J-K_{s}\right)$ diagram allows us to investigate the presence of the double red clump claimed by Mauro et al. (2012) from the analysis of VVV data. Figure 5 shows a zoom in of the red clump region, with the horizontal lines marking the magnitude limits used by Mauro et al. (2012) to separate what they named HB-A and HB-B (see their Figure 3). As is apparent, our photometry shows no strong evidence of a double $\mathrm{HB}$ in this cluster. However, a one-to-one comparison is not really possible because the VVV photometry of Mauro et al. (2012) cover a much larger FOV than the combined GEMINI$+H S T$ data but it is severely affected by crowding in the core. Instead, in agreement with Cohen et al. (2018), we confirm the presence of a blue extension of the $\mathrm{HB}$, that is somehow unexpected for a quite metal-rich GC as NGC 6569. Sequences of field stars are visible at $J-K_{s} \approx 1.0$ and run parallel to the RGB at $J-K_{s} \approx 0.6$.

The $(F 814 W, F 555 W-F 814 W)$ CMD of the cluster is shown in Figure 6. The left panel includes all stars in the WFC3 FOV, while in the right panel we show only stars located at a distance $r<r_{c}$, where $r_{c}=21^{\prime \prime}$ is the core radius of the cluster (Harris 1996). The optical CMD nicely extends for more than $10 \mathrm{mag}$, down to $F 814 W \approx 23.0$. All the evolutionary sequences are clearly distinguishable and a few blue $\mathrm{HB}$ stars at $F 814 W \approx 16.5$ can be identified also in this filter combination. Given that the WFC3 has a larger FOV than GSAOI, a larger degree of field contamination is evident and clearly appears also from the comparison of the two panels in Figure 6.

\section{Proper Motion Analysis}

To clean the observed CMDs from field star interlopers, we performed a relative PM analysis. We used the GeMS+GSAOI and HST/WFC3 data sets, which are separated by a temporal baseline of $4.419 \mathrm{yr}$, as first and second epochs, respectively. PM information from the Gaia Data Release 2 for this cluster exists only for $\sim 200$ stars along the brightest portion of the RGB and suffers from significant uncertainties.

Our analysis represents a crucial test to verify whether ground-based AO observations can be successfully combined with $H S T$ data to perform accurate PM measurements in dense stellar systems (Fritz et al. 2017 already demonstrated that they work quite well in loose environments as the halo GC Pyxis).

To measure the stellar PMs we adopted the approach described in Massari et al. (2013, see also Dalessandro et al. 2013; Bellini et al. 2014; Massari et al. 2015; Cadelano et al. 2017). Briefly, the procedure consists of measuring the displacement of the centroids of the stars measured in the two epochs, once a common reference frame is defined. The first step is to adopt a distortion-free reference frame, which we call master frame hereafter. We defined as master frame the catalog obtained from the combination of all the (second-epoch) WFC3 single-exposure catalogs, after correction for geometric distortions applied following Bellini et al. (2011). Our master frame contains only stars observed in all the available images (six for each filter). To derive accurate transformations between the first-epoch and the master catalog, we selected a sample of $\sim 3300$ bona-fide stars having magnitude $16.5 \leqslant m_{F 814 W} \leqslant 19.5$ (corresponding to magnitudes $15.0 \leqslant m_{K s} \leqslant 18.0$ ), which are likely cluster members on the basis of their CMD position (stars distributed along the lower RGB, the SGB, and the upper MS), as shown in Figure 7. We then applied a six-parameter linear transformation between 


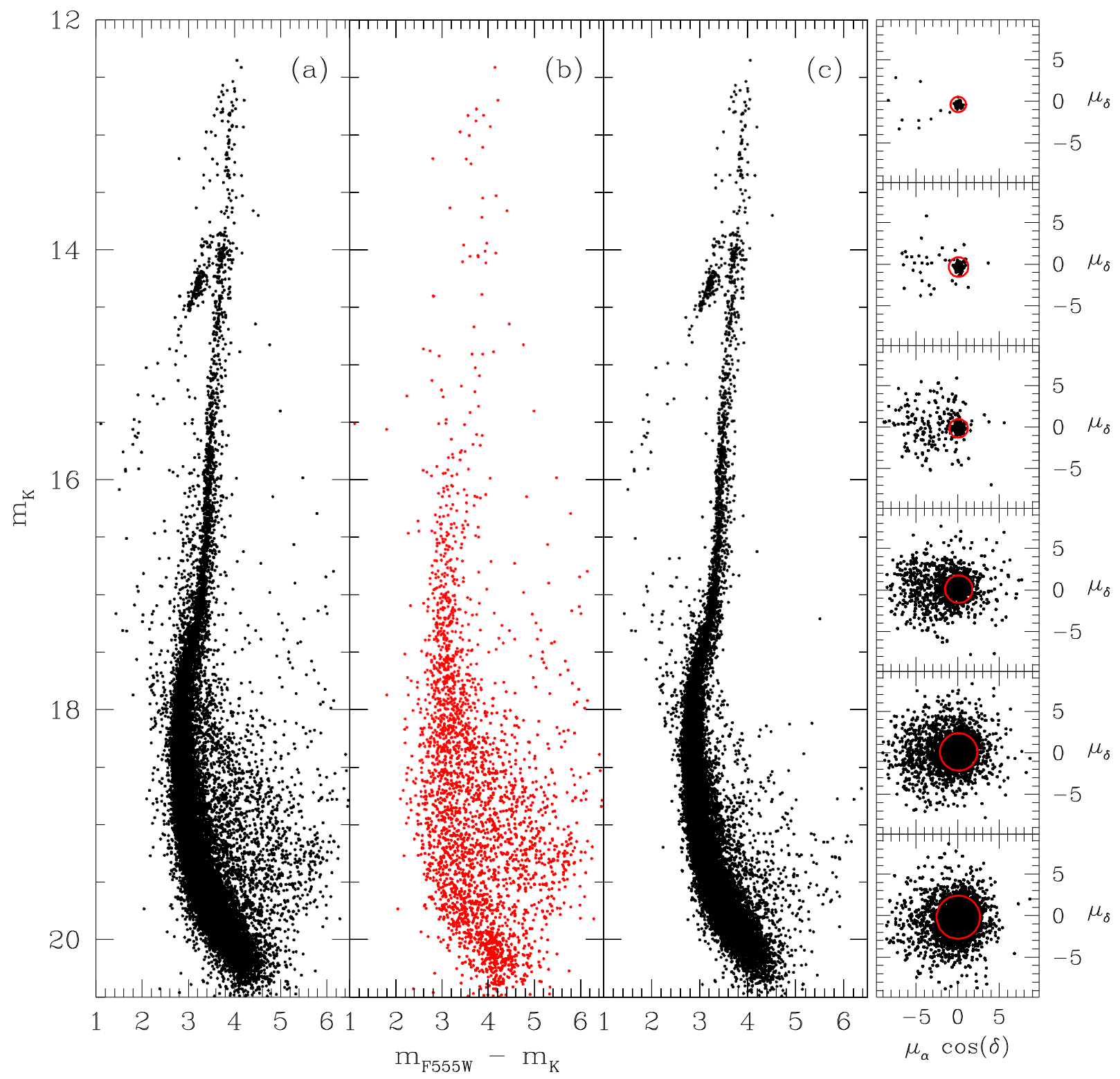

Figure 8. Panel (a): $\left(m_{K}, m_{F 555 W}-m_{K}\right)$ CMD of the stars in common between GEMINI and HST. Panel (b): CMD made of all the contaminating objects, selected from the VPDs as those with PMs not compatible with that of the GC. Panel (c): PM-cleaned CMD obtained by using only the likely cluster members selected from the VPDs shown in the rightmost column. As can be seen, sharper and better defined evolutionary sequences are now appreciable. Rightmost column: VPDs of the measured stars divided in six bins of $1.5 \mathrm{mag}$ each. The solid red circles contain the stars classified as likely cluster members.

the two epochs, determined by using the stars in common between the GSAOI and the reference stars in the master catalog. ${ }^{14}$ We treated each chip separately, in order to maximize the accuracy. Moreover, we carefully corrected the first-epoch GEMINI catalog for the important geometric distortions affecting the GSAOI camera (Ammons et al. 2016; Dalessandro et al. 2016; Massari et al. 2016b; Neichel et al. 2016; Kerber et al. 2019). To do that, we applied the geometric distortions solutions published in Dalessandro et al. (2016).

At the end of this step, for the $\sim 3300$ selected stars, we have up to 10 position measurements in the first-epoch catalog and up to 12 in the second-epoch catalog. The mean $X$ and $Y$ positions of each star in each epoch is computed by adopting a

\footnotetext{
${ }^{14}$ For the determination of both PMs and differential reddening (see Section 5) we considered only the FOV in common between the GEMINI observations and the WFC3 chip that contains the cluster center (see Figure 2).
}

$\sigma$-clipping rejection. The derived transformations have been applied to all the stars detected in each frame, by following the same procedure. The relative PM is finally determined by measuring the difference of the mean $X$ and $Y$ positions of the same star in the two epochs, divided by their temporal baseline $\Delta T=4.419 \mathrm{yr}$. Such displacements are in units of pixels $\mathrm{yr}^{-1}$.

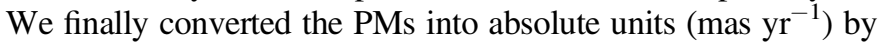
multiplying the measured displacements by the pixel scale of the master frame (0".04/pixel). On the basis of the measured PMs, we iterated the procedure by refining the master frame used to compute the six-parameter linear transformation. In particular we selected only stars with PMs $(d x, d y)<0.3$ mas yr $^{-1}$ and having magnitude $15.5 \leqslant m_{F 814 W} \leqslant 21.5$. At the end we derived relative PMs for $\sim 20,000$ individual stars in the cluster core.

Figure 8 shows the derived vector point diagrams (VPDs) and the effect of decontaminating the CMD of NGC 6569 from field stars. The hybrid $\left(m_{K}, m_{F 555 W}-m_{K}\right)$ CMD of the stars in 


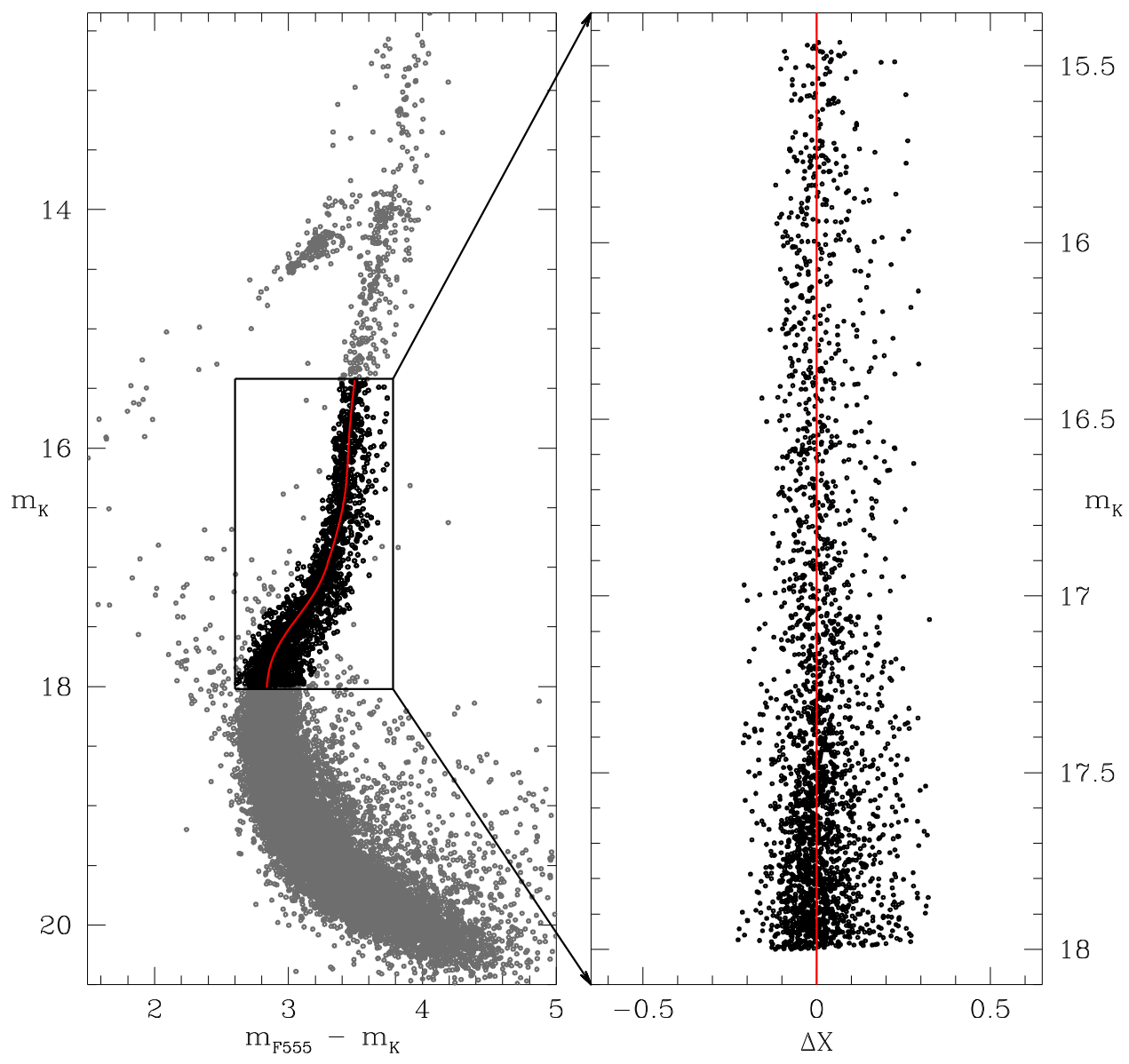

Figure 9. Left panel: the PM-corrected NIR-optical CMD of NGC 6569. The fiducial cluster MRL for $15.5 \leqslant m_{K} \leqslant 18.0$ is drawn in red and all the stars used for the differential reddening calculation are encircled by the black box and shown as black dots. Right panel: reference stars used for the differential reddening determination (those shown in black in the left panel) plotted in a rectified plane, where the $x$-axis represents the color difference of each star from the MRL, which has $\Delta X=0$ by definition.

common between $H S T$ and GEMINI is presented in the panel (a), before PM selection. The relative PM measurements are shown in the rightmost panels for six different bins of $1.5 \mathrm{mag}$ each. In each magnitude interval, we adopted a $2 \sigma$-clipping procedure to separate cluster members from field stars, where $\sigma$ is the dispersion in the VPD. The size of the plotted red circles is proportional to the value of $\sigma$ obtained after convergence. Stars within the red circles are plotted as black points in panel (c) of Figure 8, while the stars located outside the circles are shown in the panel (b). As is apparent in panel (c) of Figure 8, all the cluster evolutionary sequences are much better defined after removal of field star interlopers. The contamination observed at the MS level is mostly due to Galactic disk stars, while the narrow sequence of stars observed running parallel on the left of the RGB of NGC 6569 is likely due to bulge field stars. Interestingly, the handful of blue HB stars located at $\left(F 555 W-K_{s}\right) \approx 1.5$ in the hybrid optical-NIR CMD survived the PM cleaning, thus indicating they are likely cluster members. Also a well defined sequence of Blue Straggler Stars (Sandage 1953; Ferraro et al. 1992, 1995 ) is now clearly distinguishable in the CMD. These stars have been found to be powerful tracers of the dynamical evolution of GCs (see Ferraro et al. 2009a, 2012, 2018; Lanzoni et al. 2016): their properties will be discussed in a forthcoming paper.
Our approach demonstrates that ground-based AO observations can be successfully used to obtain accurate PMs in dense environments ${ }^{15}$ (see also Kerber et al. 2019).

\section{Differential Reddening}

Even after applying the relative PM selection presented in Section 4, the main evolutionary sequences in the CMD are rather broad compared to the photometric error measured at those levels. This is likely due to differential reddening in the sampled FOV. In fact, as already discussed in Section 1, NGC 6569 has a quite large color excess $E(B-V) \approx 0.53$ (Harris 1996) and because of its location in the Galactic bulge, it can be affected by significant differential reddening even on the small scales of our observations. We determined the differential reddening correction for all the optical $(F 555 W$ and $F 814 W)$ and NIR $\left(J\right.$ and $\left.K_{S}\right)$ filters, by using only the stars that survived the PM selection. We focused on the hybrid $\left(m_{K}, m_{F 555 W}-m_{K}\right)$ CMD for two main reasons: (1) the difference $m_{F 555 W}-m_{K}$ is the largest color baseline we can sample with the available filters (this is important to amplify the differential reddening), and

\footnotetext{
15 The approach presented here is similar to that of Massari et al. (2016b) for NGC 6681 and of Monty et al. (2018) for NGC 2298 and NGC 3201, but in those cases relative PM measurements were already available from previous works using HST (Massari et al. 2013; Simunovic \& Puzia 2016; Soto et al. 2017).
} 


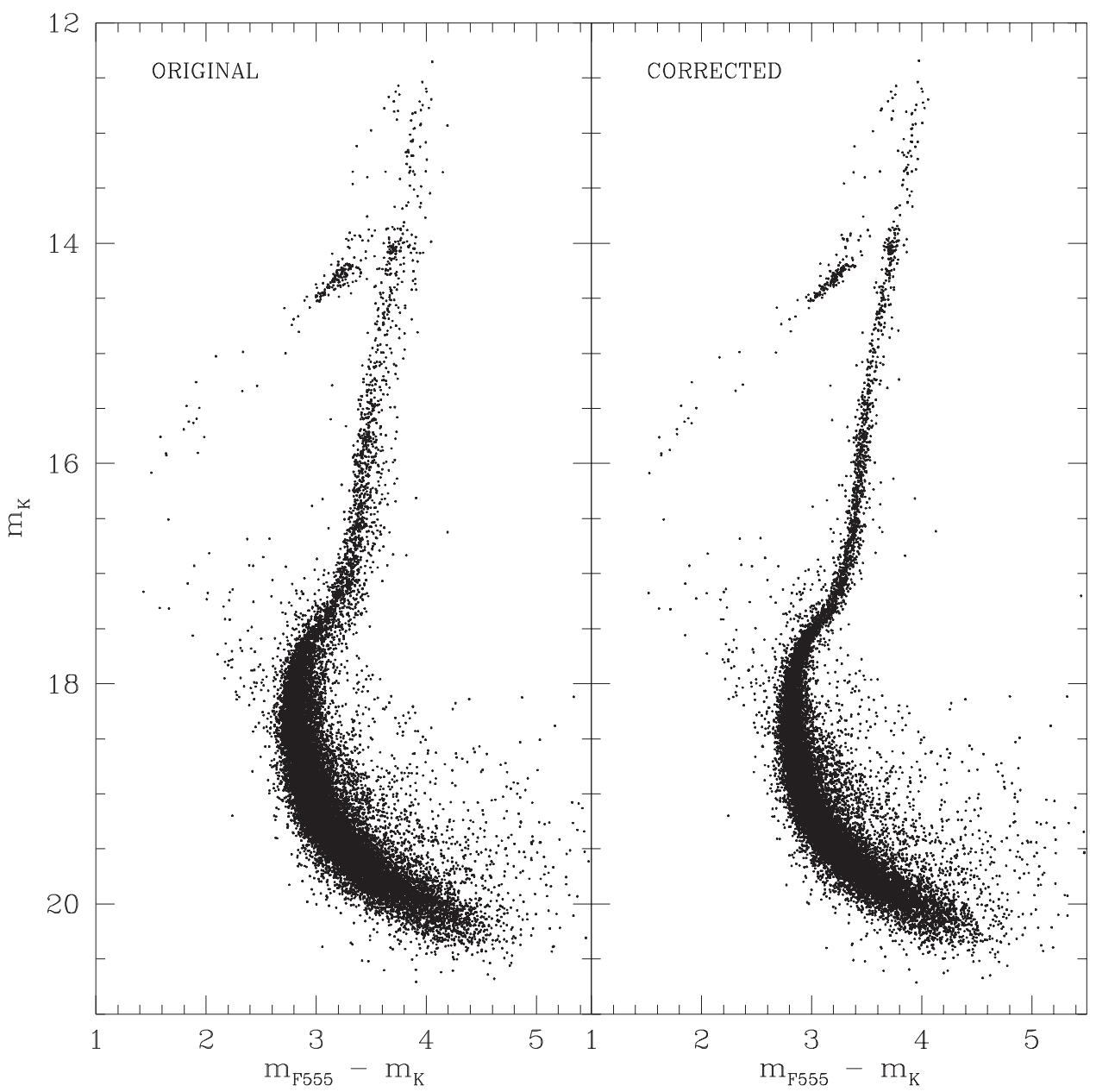

Figure 10. PM-cleaned $\left(m_{K}, m_{F 555 W}-m_{K}\right)$ CMD of NGC 6569 for the stars in common between GEMINI and HST is shown before (left panel) and after (right panel) the differential reddening correction. In the differential reddening-corrected CMD all the evolutionary sequences are better defined.

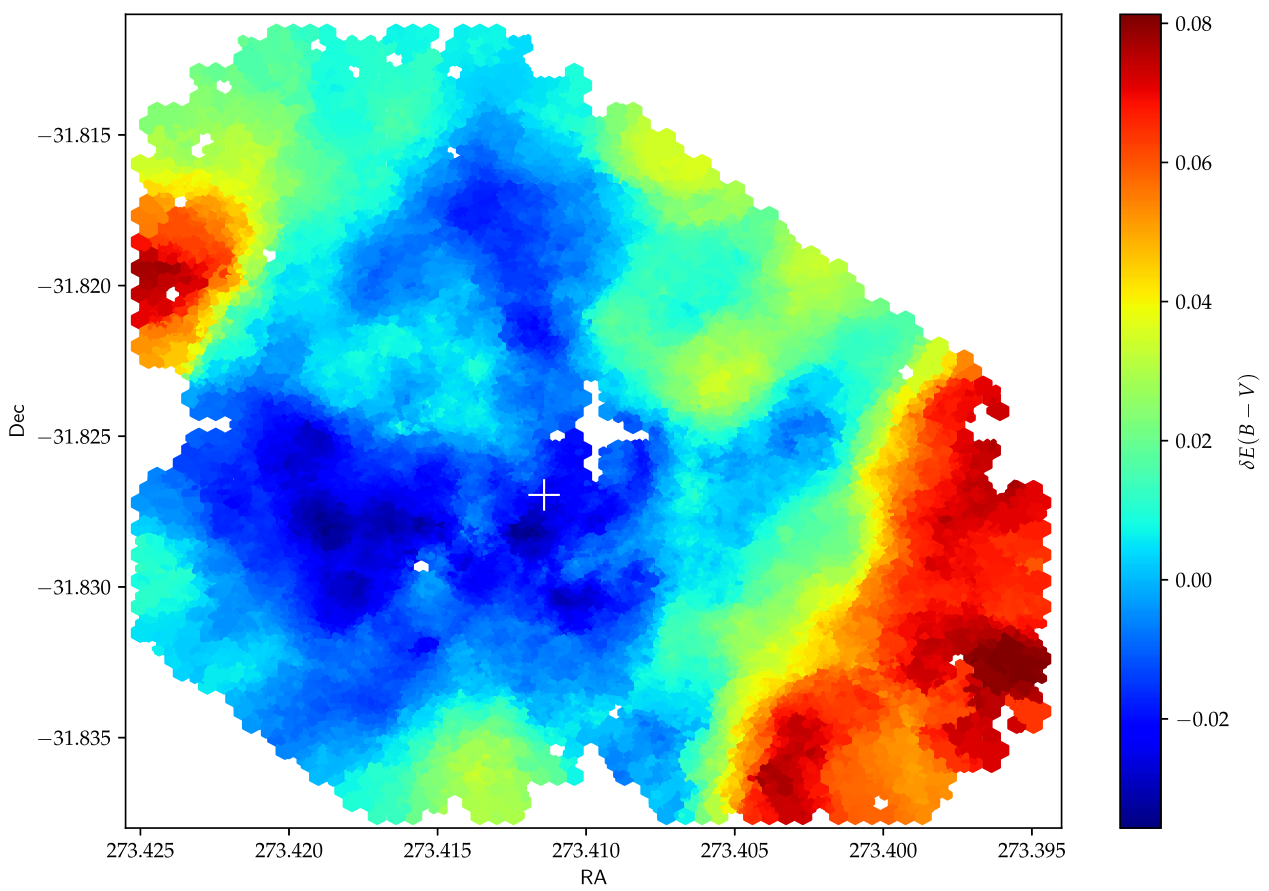

Figure 11. Differential reddening map of NGC 6569 in the GEMINI $+H S T$ FOV. The FOV has been binned in cells of $\sim 2$ square arcsec, and the $\delta E(B-V)$ value of each cell has been obtained by averaging the color excesses of the stars included. Different colors correspond to different values of $\delta E(B-V)$, as coded in the adjacent color bar. A clumpy structure is apparent. White cells correspond to saturated stars and/or uncovered regions, while the white cross marks the position of the cluster center. 


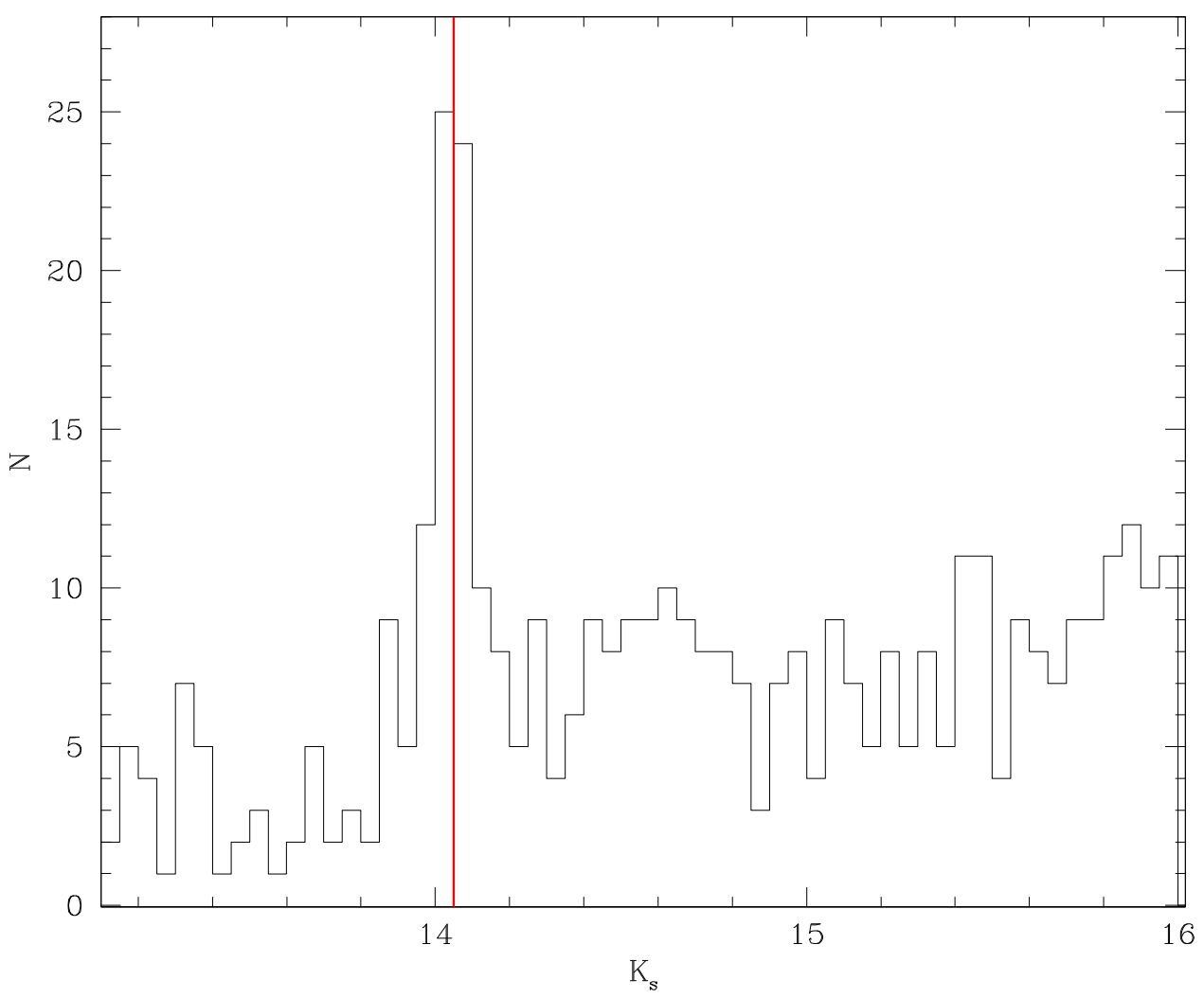

Figure 12. Luminosity function of the bright RGB stars in NGC 6569. The red vertical line marks the location of the RGB-bump.

(2) these effects are significantly smaller in $K_{s}$ than in $F 555 \mathrm{~W}$ (thus, at a first approximation, they mostly produce color shifts in the hybrid plane).

In order to estimate the differential reddening we first derived the cluster mean ridge-line (MRL) in the magnitude range $15.5 \leqslant m_{K} \leqslant 18.0$ (see Figure 9, left panel). To this end, we divided the considered portion of the CMD in different magnitude bins, and in each of them we computed the mean color after a $2 \sigma$-clipping rejection. We adopted five different bin widths, ranging from 0.2 to 0.4 mag by steps of 0.05 mag. In each of these five realizations an MRL was obtained. The final MRL is then given by the average of the five values after a resampling with a cubic spline of 0.01 mag steps (red line in Figure 9, left panel). For all the stars used to derive the MRL (black dots in Figure 9, left panel) we then computed the geometrical distance $(\Delta X)$ to the MRL. The distribution of $\Delta X$ as a function of magnitude for the reference sample is shown in the right panel of Figure 9. This reference sample has finally been used to assign a $\Delta X$ value to all the stars in our photometric catalog: for every source, $\Delta X$ has been determined as the average, with a $2 \sigma$-clipping rejection, of the $\Delta X$ values measured for the 20 spatially closest reference stars. ${ }^{16}$ The observed $\Delta X$ can then be easily transformed into the local differential reddening, $\delta E(B-V)$, by using the following equation:

$$
\delta E(B-V)=\frac{\Delta X}{R_{F 555 W}-R_{K}},
$$

\footnotetext{
16 This number can be considered as the best compromise between having good statistics and a good spatial resolution. The result does not change significantly if a slightly different number of nearest reference stars (from 10 to 30) is adopted.
}

where the adopted extinction coefficients are $R_{F 555 W}=3.274$ and $R_{K}=0.366$ (see Table A1 in Casagrande \& VandenBerg 2014). The $\delta E(B-V)$ values were then applied to all the available filters. We show in Figure 10 the $\left(m_{K}, m_{F 555 W}-m_{K}\right)$ CMD of NGC 6569 before (left), and after the differential reddening correction (right). The differential reddening map of NGC 6569 in the overlapping GEMINI and HST FOVs is shown in Figure 11. In this region $\delta E(B-V)$ varies by $\sim 0.12 \mathrm{mag}$, from -0.036 up to 0.081 , with a clumpy structure. The right panel of Figure 10 shows the nice CMD obtained after the differential reddening correction (and PM cleaning). All the main evolutionary sequences are now much better defined. We confirm that, after these corrections, the cluster unambiguously shows an HB morphology with a single red clump and a quite extended tail toward blue colors.

\section{Distance Determinations to NGC 6569}

An estimate of the distance modulus of NGC 6569 can be derived from the RR Lyrae stars that belong to the cluster. To this purpose, we used the mean $V$ magnitude $(\langle V\rangle=17.36)$ of the 25 RR Lyrae presented in the OGLE catalog and the very recent $M_{V}-[\mathrm{Fe} / \mathrm{H}]$ relations calibrated for this type of star from the Gaia Collaboration et al. (2017) based on the Tycho-Gaia Astrometric Solution. Since three different methods have been used to perform such a calibration, we adopted the mean $\left\langle M_{V}\right\rangle$ solution and three main sources of uncertainties: $(1) \sim 0.10$ dex in $[\mathrm{Fe} / \mathrm{H}],(2)$ the stochastic effects in the fits $(0.04 \mathrm{mag})$, (3) and the systematics due to the different solutions $(0.10 \mathrm{mag})$. With the assumed metallicity $[\mathrm{Fe} / \mathrm{H}]=-0.87$, we obtain $\left\langle M_{V}\right\rangle=0.72 \pm 0.11$ for the considered $25 \mathrm{RR}$ Lyrae stars and 

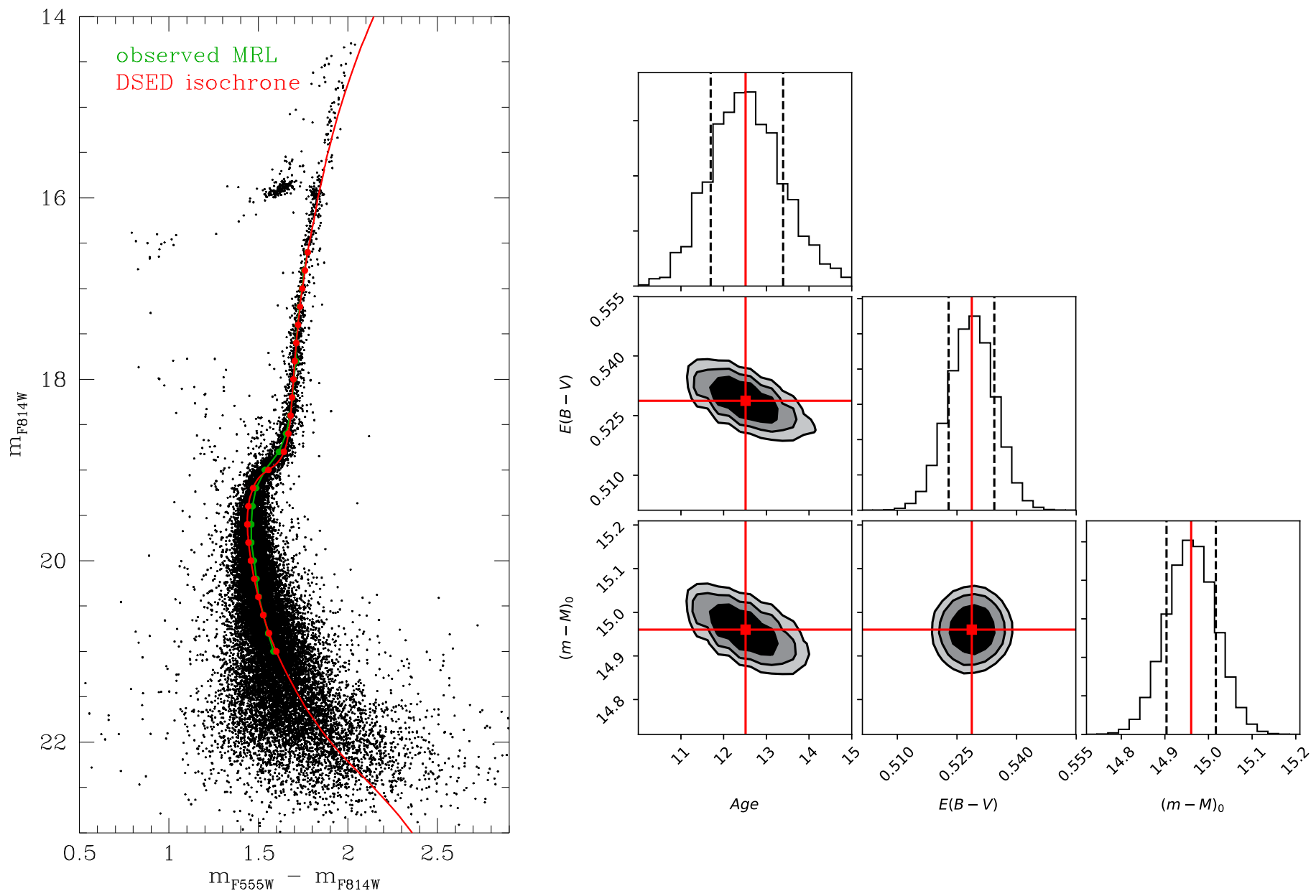

Figure 13. Left panel: the fiducial line of NGC 6569 is superimposed in dark green on the optical CMD of NGC 6569. In addition, the best-fit solution for the DSED set of isochrones is shown as a red line. The best-fit parameter values are presented in Table 1, left column. The green and red points mark, respectively, the observed and the theoretical color values used to measure the $\chi^{2}$. Right panel: corner plots showing the one- and two-dimensional projections of the posterior probability distributions for all the parameters derived from the MCMC method for the DSED isochrones. The contours correspond to the $1 \sigma, 2 \sigma$, and $3 \sigma$ levels.

thus a distance modulus in the $V$ band $(m-M)_{V}=16.64 \pm$ 0.11 . By assuming $R_{V}=3.1$ as extinction coefficient in the $V$ band, the true distance modulus of NGC 6569 becomes $(m-M)_{0}=15.00 \pm 0.11$. This value varies by only \pm 0.01 mag when the subsample of seven RR Lyrae stars in common between our PM-cleaned HST data set and the OGLE catalog (Soszyński et al. 2017) is considered.

The right panel of Figure 10 also shows a well defined clump of stars slightly brighter than the red clump along the now narrow RGB: this is the so-called RGB-bump, an evolutionary feature that flags the moment the H-burning shell reaches the $\mathrm{H}$ discontinuity left from the inner penetration of the convective envelope (see Fusi Pecci et al. 1990; Ferraro et al. 1999, 2000). Figure 12 shows the differential luminosity function of the bright RGB stars in the $K_{s}$ band, where the RGB-bump can be easily detected at $K_{s}=14.05 \pm 0.05 \mathrm{mag}$. This feature was identified in the NIR CMDs of 24 Galactic GCs by Valenti et al. (2004), who provided a relation linking the absolute $K_{s}$ magnitude of the RGB-bump and the global cluster metallicity $[\mathrm{M} / \mathrm{H}]: M_{K}=-0.17+2.07[\mathrm{M} / \mathrm{H}]+0.49[\mathrm{M} / \mathrm{H}]^{2}$. Since the metallicity of NGC 6569 is well constrained both in terms of iron and $\alpha$-elements, this relation can be used for an independent estimate of the cluster distance modulus. By adopting $[\mathrm{Fe} / \mathrm{H}]=-0.87$ and $[\alpha / \mathrm{Fe}]=+0.4$ and the relation between the iron abundance and the global metallicity presented in Salaris et al. (1993), we obtain $[\mathrm{M} / \mathrm{H}]=-0.58$. From the Valenti et al. (2004) relation we thus obtain $M_{K}=-1.21 \pm 0.12$, from which the distance modulus in the $K_{s}$ band can be derived: $(m-M)_{K}=15.26 \pm 0.13$. By assuming an average reddening of $E(B-V)=0.53$ (Ortolani et al. 2001) in the direction of the cluster and the extinction coefficients adopted in Section 5, the true distance modulus of NGC 6569 turns out to be $(m-M)_{0}=15.07 \pm 0.13$, in excellent agreement with the previous estimate.

The position in magnitude of the RGB-bump depends both on the metallicity and the cluster age, but the finding of consistent distance values using independent methods is proof that the age of this cluster is similar (i.e., old) to that of the GCs used by Valenti et al. (2004) for the calibration of the $M_{K}-[\mathrm{M} / \mathrm{H}]$ relation. We thus adopt the weighted mean of the two derived values as the distance modulus of NGC 6569: $(m-M)_{0}=15.03 \pm 0.08$, corresponding to $10.1 \pm 0.2 \mathrm{kpc}$ from the Sun. This is also in very good agreement with the previous determination of Ortolani et al. (2001), who quote $(m-M)_{0}=14.96 \pm 0.20$, but smaller than the distance of $10.9 \mathrm{kpc}$ given by Harris (1996).

\section{Age Estimate}

In the following analysis we use the PM-cleaned and differential reddening-corrected $\left(m_{F 814 W}, m_{F 555 W}-m_{F 814}\right)$ CMD of NGC 

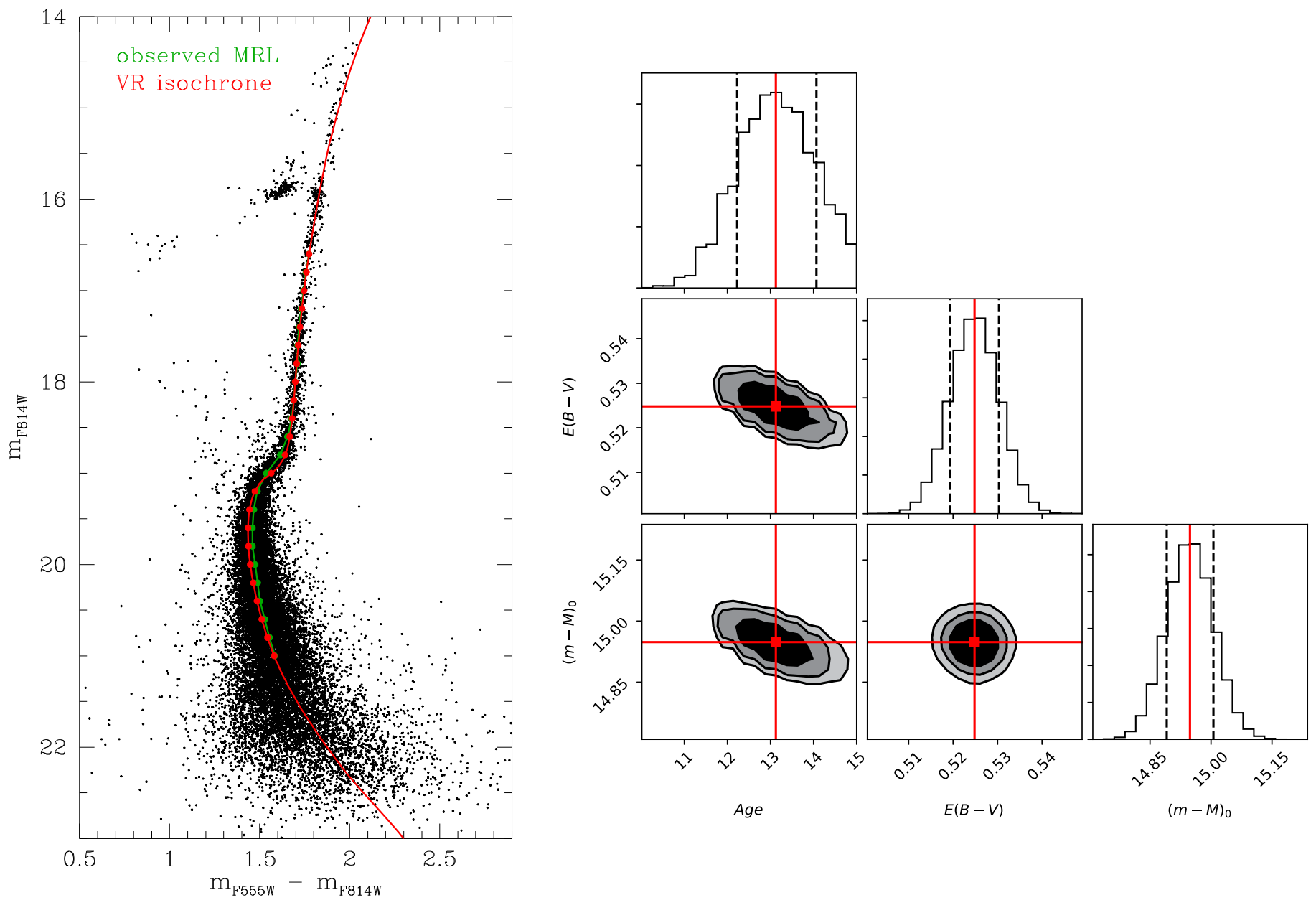

Figure 14. Similar to Figure 13 but for the VR models. The best-fit parameter values are presented in Table 1, middle column.

6569 to derive the cluster absolute age via the isochrone fitting method. The adopted approach allows us to simultaneously estimate the age, distance modulus, and color excess of the cluster through a one-to-one comparison between the observed CMD and a set of theoretical models of suitable chemical composition, exploring reasonable grids of values for each of the three parameters (see also Correnti et al. 2016, 2018; Kerber et al. 2018, 2019).

The observed CMD has been compared to three different sets of $\alpha$-enhanced isochrones: the Dartmouth Stellar Evolutionary Database (DSED, Dotter et al. 2008), the VictoriaRegina Isochrone Database (VR, VandenBerg et al. 2014) and a Bag of Stellar Tracks and Isochrones (BaSTI, Pietrinferni et al. 2004). For each family of models we assumed $[\mathrm{Fe} / \mathrm{H}]=$ $-0.87,[\alpha / \mathrm{Fe}]=+0.4$, and a helium mass fraction $Y=$ 0.2522 ( 0.253 for the BaSTI isochrones). Since no age estimates are available in the literature for NGC 6569, we explored a wide range of values appropriate for an old system, uniformly distributed from 10 to $15 \mathrm{Gyr}$, in steps of $0.5 \mathrm{Gyr}$. We then allowed the color excess $E(B-V)$ to vary by \pm 0.1 in steps of $0.02 \mathrm{mag}$ from the previously adopted value $(0.53$, Ortolani et al. 2001), assuming a uniform prior probability distribution. Finally, we allowed the distance modulus to vary by \pm 0.2 mag following a normal distribution with peak value and dispersion equal to the values obtained in the previous section (namely, 15.03 and 0.08, respectively).
From an operational point of view, we determined the MRL in the optical CMD, by measuring the mean color (after a $2 \sigma$ clipping rejection) in bins of $0.2 \mathrm{mag}$ along the main cluster evolutionary sequences, in the range $15.5 \leqslant m_{F 814} \leqslant 21.0$ where both saturation effects and photometric errors are small. In the same magnitude range, each isochrone has been resampled in steps of $0.2 \mathrm{mag}$ using a cubic spline.

To determine the best-fit parameters, as well as to study the confidence intervals and the correlations among them, we applied the Markov Chain Monte Carlo (MCMC) sampling technique. For that purpose, we used the emcee (ForemanMackey et al. 2013) code to sample the posterior probability in the three-dimensional parameter space, assuming the likelihood $\propto \exp \left(-\chi^{2} / 2\right)$.

The $\chi^{2}$ value is computed as follows:

$$
\chi^{2}=\sum_{i=1}^{N_{\text {bin }}}\left[\frac{(\text { color })_{\text {obs }, i}-(\text { color })_{\bmod , i}}{\sigma_{\text {color }, \mathrm{obs}}}\right]^{2},
$$

where $N_{\text {bin }}$ indicates the number of magnitude bins along the MRL. (color) $)_{\mathrm{obs}, i}$ and (color) $)_{\bmod , i}$ are, respectively, the observed and the theoretical $(F 555 W-F 814 W)$ mean colors in the $i$ th magnitude bin along the MRL, and $\sigma_{\text {color,obs }}$ is the standard deviation associated with the observed mean color.

The results obtained in terms of age, distance modulus, and color excess are shown in Figures 13-15 for the three adopted sets of theoretical models. 

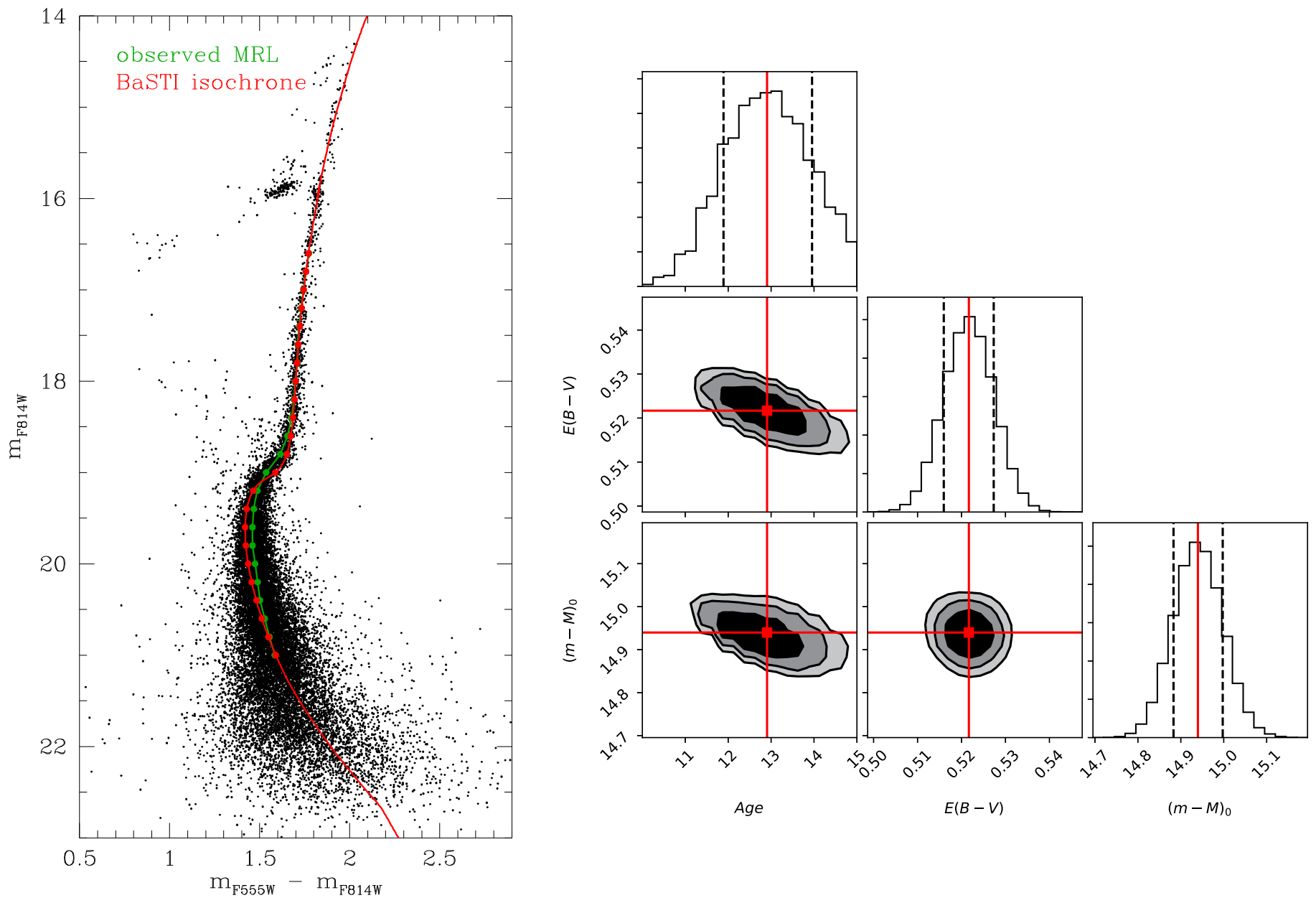

Figure 15. Similar to Figure 13 but for the BaSTI models. The best-fit parameter values are presented in Table 1, right column.

Table 1

Best-fit Parameter Values for the DSED, VR, and BaSTI Models

\begin{tabular}{lccc}
\hline \hline & DSED Isochrone & VR Isochrone & BaSTI Isochrone \\
\hline$E(B-V)[\mathrm{mag}]$ & $0.53 \pm 0.01$ & $0.525 \pm 0.010$ & $0.52 \pm 0.01$ \\
$(m-M)_{0}[\mathrm{mag}]$ & $14.96_{-0.06}^{+0.06}$ & $14.95_{-0.06}^{+0.06}$ & $14.94_{-0.06}^{+0.06}$ \\
Age $[\mathrm{Gyr}]$ & $12.51_{-0.82}^{+0.88}$ & $13.13_{-0.90}^{+0.94}$ & $12.91_{-1.01}^{+1.05}$ \\
\hline
\end{tabular}

In each figure the left-hand panel shows the observed optical CMD with the fiducial MRL drawn in green and the best-fit isochrone plotted as a red line. In all cases, the best-fit model reproduces reasonably well the cluster fiducial line along all of the evolutionary sequences in the CMD. We also verified that the lower envelope of the distribution of HB stars is also nicely reproduced up to bluer colors when the related ZAHB models are considered (Pietrinferni et al. 2004, D. VandenBerg 2018, private communication). The reduced (per degree of freedom) $\chi^{2}$ values (that are obtained from the color values marked with green and red dots in the figures) are $0.15,0.20$, and 0.48 for the DSED, VR, and BaSTI isochrones, respectively. The oneand two-dimensional posterior probabilities for all of the parameter combinations are presented in the right-hand panel of each figure as corner plots. Reddening and distance are not mutually correlated, while there is a strong degeneracy of these two parameters with age.
The best-fit values and their $1 \sigma$ uncertainties obtained for $E(B-V),(m-M)_{0}$, and age from the three sets of models are listed in Table 1 . They are all consistent within the errors. NGC 6569 turns out to be a quite old cluster, with an age of about $12.8 \mathrm{Gyr}$, and a typical age uncertainty of about $0.8-1.0$ Gyr. Differences in the best-fit age are somewhat expected when different models, which adopt slightly different solar abundances, opacities, reaction rates, efficiency of atomic diffusion, etc., are compared. We also note that an uncertainty of $\sim \pm 0.1-0.2$ Gyr has to be added to the overall error budget when the effect of a slightly different metallicity (by $\pm 0.1 \mathrm{dex}$ ) for the cluster is considered.

\subsection{Comparing NGC 6569 with Other Bulge GCs}

In this section we compare the age estimates obtained in the present work for NGC 6569 with those of other bulge GCs. ${ }^{17}$ Unfortunately, up to now, only a dozen bulge GCs have accurate age measurements, mainly because of their strong foreground extinction. The only cases are NGC 6304 (Dotter et al. 2010); NGC 6637, NGC 6652, and NGC 6723 (Dotter et al. 2010; VandenBerg et al. 2013); NGC 6522 and NGC 6626 (Kerber et al. 2018); NGC 6624 (Dotter et al. 2010; VandenBerg et al. 2013; Saracino et al. 2016); NGC 6553

\footnotetext{
17 The age estimates derived for different clusters come from slightly different approaches and/or assumptions, so that the direct comparison may be affected by some systematic uncertainties.
} 


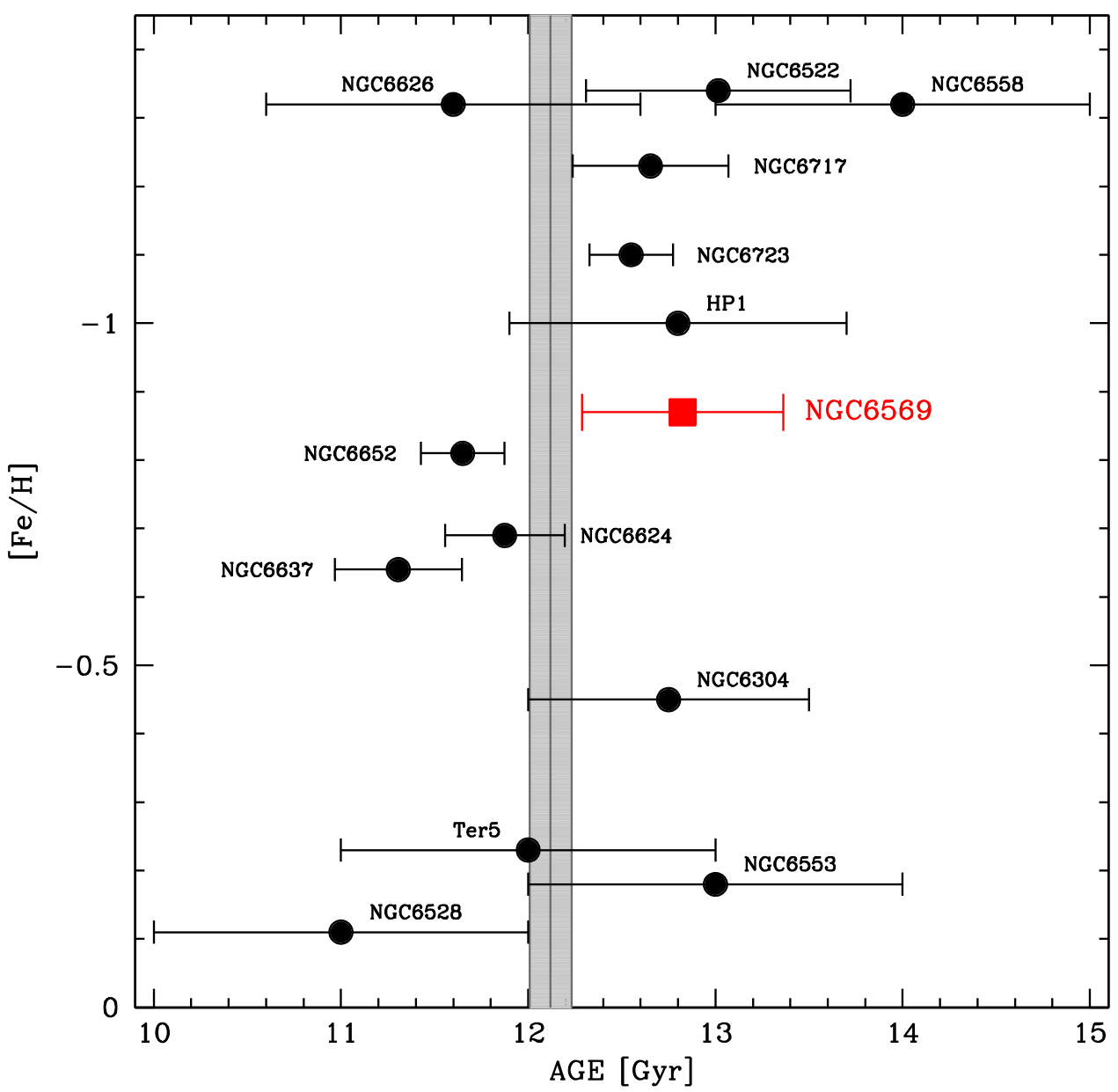

Figure 16. Absolute age of NGC 6569 (red square) derived in this work as the weighted mean of the determinations given by the three sets of models listed in Table 1 is compared to that obtained for other bulge GCs in the literature (black circles; see references in Section 7.1), as a function of their metallicity. Different age estimates for a given system have been averaged in order to have a single value for each cluster. The vertical gray strip marks the weighted mean of the entire sample and its $1 \sigma$ uncertainty.

(Zoccali et al. 2001); NGC 6528 (Lagioia et al. 2014; Calamida et al. 2014); NGC 6558 (Barbuy et al. 2007); and Terzan 5 (Ferraro et al. 2016; here we take into account only the old, dominant component of the system) and HPl (Kerber et al. 2019). The current situation is summarized in Figure 16, where we plot the age estimates currently available for bulge GCs, as a function of their metallicity (from Harris 1996 with only two exceptions: NGC 6569, Johnson et al. 2018 and NGC 6624, Valenti et al. 2011). For those systems for which more than one age value is quoted in the literature, we plot the weighted means and relative errors. All bulge GCs are old, with ages in excess of $\approx 11 \mathrm{Gyr}$, independently of their metallicity. The weighted mean age of the entire sample is $12.1 \pm 0.1 \mathrm{Gyr}$, as marked by the vertical gray strip in Figure 16. Such an old age of the bulge GC system is consistent with that of bulge stars observed in different fields (Zoccali et al. 2003; Clarkson et al. 2011; Valenti et al. 2013), confirming that the stellar populations in the innermost regions of the Milky Way were born at the earliest epochs of the Galaxy's formation.

\section{Summary and Conclusions}

By using the high-resolution imaging cameras WFC3 on board HST in the optical and GeMS/GSAOI at GEMINI in the NIR, we obtained deep and accurate photometry in different filter combinations of the bulge GC NGC 6569. The derived
CMDs span a range of about 8 mag, allowing us to identify all the well known evolutionary sequences, from the HB level down to about two magnitudes below the MS-TO.

For the first time we determined relative PMs for about 20,000 individual stars in this cluster, sampling from the bright RGB to the faint MS. To this end, we used GEMINI and HST observations as first- and second-epoch data, respectively.

We also determined the first differential reddening map in the direction of the system, finding that the color excess $E(B-V)$ varies by $\sim 0.12 \mathrm{mag}$ across the sampled FOV and it has a clumpy distribution. By using the observed position of the RGB-bump and the average $V$-band magnitude of a sample of 25 RR Lyrae stars, we find that NGC 6569 has a distance modulus $(m-M)_{0}=15.03 \pm 0.08$, corresponding to $10.1 \pm$ $0.2 \mathrm{kpc}$ from the Sun.

Finally, by taking advantage of our high-resolution PMcleaned and differential reddening-corrected CMD we obtained the first accurate estimate of the absolute age of NGC 6569, The isochrone fitting method, based on a $\chi^{2}$ statistics, has been used to compare three different sets of theoretical models (DSED, $\mathrm{VR}$, and BaSTI) with the observed $\left(m_{F 814 W}, m_{F 555 W}-m_{F 814 W}\right)$ CMD of the cluster. By adopting as input "guess" values the distance modulus determined in this work and the reddening quoted in the literature (0.53, from Ortolani et al. 2001), we find that NGC 6569 has an absolute age of $12.8 \pm 1.0 \mathrm{Gyr}$. We also 
confirmed the presence of a prominent, single red clump and of some blue HB stars.

The promising results obtained in this study confirm that ground-based NIR imaging assisted by MCAO as provided by GeMS/GSAOI can be efficiently used for deep and accurate photometric and astrometric analysis of dense stellar fields in the central region of the Galaxy severely affected by extinction, once the geometric distortions of the detectors are properly corrected.

We thank the anonymous referee for a careful reading of the paper and for helping us to improve the presentation of our work. S.S. is grateful to Don Vandenberg for computing and sharing the Victoria-Regina ZAHB models used in the paper for comparison. D.G. gratefully acknowledges support from the Chilean Centro de Excelencia en Astrofísica y Tecnologías Afines (CATA) BASAL grant AFB-170002. D.G. also acknowledges financial support from the Dirección de Investigación y Desarrollo de la Universidad de La Serena through the Programa de Incentivo a la Investigación de Académicos (PIA-DIDULS).

Software: emcee (Foreman-Mackey et al. 2013), CataXcorr, IRAF (Tody 1986, 1993), DAOPHOTIV (Stetson 1987, 1994), ALLFRAME (Stetson 1994).

\section{ORCID iDs}

S. Saracino (1D https://orcid.org/0000-0003-4746-6003

E. Dalessandro (iD https://orcid.org/0000-0003-4237-4601

F. R. Ferraro (iD https://orcid.org/0000-0002-2165-8528

B. Lanzoni (i) https://orcid.org/0000-0001-5613-4938

D. Geisler (D) https://orcid.org/0000-0002-3900-8208

A. Bellini (i) https://orcid.org/0000-0003-3858-637X

S. Cassisi (iD https://orcid.org/0000-0001-5870-3735

L. Origlia (1) https://orcid.org/0000-0002-6040-5849

S. Villanova (iD https://orcid.org/0000-0001-6205-1493

\section{References}

Ammons, S. M., Garcia, E. V., Salama, M., et al. 2016, Proc. SPIE, 9909, 99095T

Anderson, J., \& van der Marel, R. P. 2010, ApJ, 710, 1032

Barbuy, B., Chiappini, C., \& Gerhard, O. 2018, ARA\&A, 56, 223

Barbuy, B., Zoccali, M., Ortolani, S., et al. 2007, AJ, 134, 1613

Barnard, E. E., Frost, E. B., \& Calvert, M. R. 1927, A Photographic Atlas of Selected Regions of the Milky Way (Washington, DC: Carnegie Institution) Bellini, A., Anderson, J., \& Bedin, L. R. 2011, PASP, 123, 622

Bellini, A., Anderson, J., van der Marel, R. P., et al. 2014, ApJ, 797, 115 Bica, E., Bonatto, C., Barbuy, B., \& Ortolani, S. 2006, A\&A, 450, 105 Cadelano, M., Dalessandro, E., Ferraro, F. R., et al. 2017, ApJ, 836, 170 Calamida, A., Bono, G., Lagioia, E. P., et al. 2014, A\&A, 565, A8 Casagrande, L., \& VandenBerg, D. A. 2014, MNRAS, 444, 392 Clarkson, W. I., Sahu, K. C., Anderson, J., et al. 2011, ApJ, 735, 37 Cohen, R. E., Mauro, F., Alonso-García, J., et al. 2018, AJ, 156, 41 Cohen, R. E., Moni Bidin, C., Mauro, F., Bonatto, C., \& Geisler, D. 2017, MNRAS, 464, 1874

Correnti, M., Gennaro, M., Kalirai, J. S., Brown, T. M., \& Calamida, A. 2016, ApJ, 823, 18

Correnti, M., Gennaro, M., Kalirai, J. S., Cohen, R. E., \& Brown, T. M. 2018, ApJ, 864, 147

Dalessandro, E., Ferraro, F. R., Massari, D., et al. 2013, ApJ, 778, 135

Dalessandro, E., Pallanca, C., Ferraro, F. R., et al. 2014, ApJL, 784, L29

Dalessandro, E., Saracino, S., Origlia, L., et al. 2016, ApJ, 833, 111

Dotter, A., Chaboyer, B., Jevremović, D., et al. 2008, ApJS, 178, 89

Dotter, A., Sarajedini, A., Anderson, J., et al. 2010, ApJ, 708, 698

Ferraro, F. R., Beccari, G., Dalessandro, E., et al. 2009a, Natur, 462, 1028
Ferraro, F. R., Clementini, G., Fusi Pecci, F., \& Buonanno, R. 1991, MNRAS, 252, 357

Ferraro, F. R., Dalessandro, E., Mucciarelli, A., et al. 2009b, Natur, 462, 483

Ferraro, F. R., Fusi Pecci, F., \& Bellazzini, M. 1995, A\&A, 294, 80

Ferraro, F. R., Fusi Pecci, F., \& Buonanno, R. 1992, MNRAS, 256, 376

Ferraro, F. R., Lanzoni, B., Dalessandro, E., et al. 2012, Natur, 492, 393

Ferraro, F. R., Lanzoni, B., Raso, S., et al. 2018, ApJ, 860, 36

Ferraro, F. R., Massari, D., Dalessandro, E., et al. 2016, ApJ, 828, 75

Ferraro, F. R., Messineo, M., Fusi Pecci, F., et al. 1999, AJ, 118, 1738

Ferraro, F. R., Montegriffo, P., Origlia, L., \& Fusi Pecci, F. 2000, AJ, 119,1282

Ferraro, F. R., Pallanca, C., Lanzoni, B., et al. 2015, ApJL, 807, L1

Foreman-Mackey, D., Hogg, D. W., Lang, D., \& Goodman, J. 2013, PASP, 125,306

Fritz, T. K., Linden, S. T., Zivick, P., et al. 2017, ApJ, 840, 30

Fusi Pecci, F., Ferraro, F. R., Crocker, D. A., Rood, R. T., \& Buonanno, R. 1990, A\&A, 238, 95

Gaia Collaboration, Clementini, G., Eyer, L., et al. 2017, A\&A, 605, A79

Harris, W. E. 1996, AJ, 112, 1487

Hazen-Liller, M. L. 1984, BAAS, 16, 967

Hazen-Liller, M. L. 1985, AJ, 90, 180

Johnson, C. I., Rich, R. M., Caldwell, N., et al. 2018, AJ, 155, 71

Kerber, L. O., Libralato, M., Souza, S. O., et al. 2019, MNRAS, 484, 5530

Kerber, L. O., Nardiello, D., Ortolani, S., et al. 2018, ApJ, 853, 15

Kunder, A., Stetson, P. B., Catelan, M., et al. 2015, in ASP Conf. Ser. 491, Fifty Years of Wide Field Studies in the Southern Hemisphere: Resolved Stellar Populations of the Galactic Bulge and Magellanic Clouds, ed. S. Points \& A. Kunder (San Francisco, CA: ASP), 104

Lagioia, E. P., Milone, A. P., Stetson, P. B., et al. 2014, ApJ, 782, 50

Lanzoni, B., Ferraro, F. R., Alessandrini, E., et al. 2016, ApJL, 833, L29

Lanzoni, B., Ferraro, F. R., Dalessandro, E., et al. 2010, ApJ, 717, 653

Libralato, M., Bellini, A., van der Marel, R. P., et al. 2018, ApJ, 861, 99

Massari, D., Bellini, A., Ferraro, F. R., et al. 2013, ApJ, 779, 81

Massari, D., Dalessandro, E., Ferraro, F. R., et al. 2015, ApJ, 810, 69

Massari, D., Fiorentino, G., McConnachie, A., et al. 2016a, A\&A, 586, A51

Massari, D., Fiorentino, G., McConnachie, A., et al. 2016b, A\&A, 595, L2

Massari, D., Mucciarelli, A., Dalessandro, E., et al. 2012, ApJL, 755, L32

Massari, D., Mucciarelli, A., Ferraro, F. R., et al. 2014a, ApJ, 791, 101

Massari, D., Mucciarelli, A., Ferraro, F. R., et al. 2014b, ApJ, 795, 22

Mauro, F., Moni Bidin, C., Cohen, R., et al. 2012, ApJL, 761, L29

McLaughlin, D. E., Anderson, J., Meylan, G., et al. 2006, ApJS, 166, 249

McNamara, B. J., Harrison, T. E., Baumgardt, H., \& Khalaj, P. 2012, ApJ, 745,175

Melcher, H., \& Gerth, E. 1977, Exp. Tech. Phys., 25, 527

Moffat, A. F. J. 1969, A\&A, 3, 455

Monty, S., Puzia, T. H., Miller, B. W., et al. 2018, arXiv:1808.05271

Neichel, B., Fusco, T., Sauvage, J.-F., et al. 2016, Proc. SPIE, 9909 990909

Neichel, B., Rigaut, F., Vidal, F., et al. 2014, MNRAS, 440, 1002

Ness, M., Freeman, K., Athanassoula, E., et al. 2013, MNRAS, 430, 836

Origlia, L. 2014, in IAU Symp. 298, Setting the Scene for Gaia and LAMOST (Cambridge: Cambridge Univ. Press), 28

Origlia, L., Massari, D., Rich, R. M., et al. 2013, ApJL, 779, L5

Origlia, L., Rich, R. M., Ferraro, F. R., et al. 2011, ApJL, 726, L20

Ortolani, S., Barbuy, B., Momany, Y., et al. 2011, ApJ, 737, 31

Ortolani, S., Bica, E., \& Barbuy, B. 2001, A\&A, 374, 564

Penny, A. J. 1976, PhD Thesis, Sussex Univ.

Pietrinferni, A., Cassisi, S., Salaris, M., \& Castelli, F. 2004, ApJ, 612, 168

Piotto, G., King, I. R., Djorgovski, S. G., et al. 2002, A\&A, 391, 945

Rich, R. M. 1998, in IAU Symp. 184, The Central Regions of the Galaxy and Galaxies, ed. Y. Sofue (Dordrecht: Kluwer), 11

Rosino, L. 1962, MmSAI, 33, 351

Salaris, M., Chieffi, A., \& Straniero, O. 1993, ApJ, 414, 580

Sandage, A. R. 1953, AJ, 58, 61

Saracino, S., Dalessandro, E., Ferraro, F. R., et al. 2015, ApJ, 806, 152

Saracino, S., Dalessandro, E., Ferraro, F. R., et al. 2016, ApJ, 832, 48

Saracino, S., Dalessandro, E., Ferraro, F. R., et al. 2018, ApJ, 860, 95

Simunovic, M., \& Puzia, T. H. 2016, MNRAS, 462, 3401

Soszyński, I., Udalski, A., Szymański, M. K., et al. 2017, AcA, 67, 297

Soto, M., Bellini, A., Anderson, J., et al. 2017, AJ, 153, 19

Stetson, P. B. 1987, PASP, 99, 191

Stetson, P. B. 1994, PASP, 106, 250 
Tody, D. 1986, Proc. SPIE, 627, 733

Tody, D. 1993, in ASP Conf. Ser. 52, Astronomical Data Analysis Software and Systems II, ed. R. J. Hanisch, R. J. V. Brissenden, \& J. Barnes (San Francisco, CA: ASP), 173

Trager, S. C., King, I. R., \& Djorgovski, S. 1995, AJ, 109, 218

Valenti, E., Ferraro, F. R., \& Origlia, L. 2004, MNRAS, 354, 815

Valenti, E., Ferraro, F. R., \& Origlia, L. 2007, AJ, 133, 1287

Valenti, E., Origlia, L., \& Ferraro, F. R. 2005, MNRAS, 361, 272

Valenti, E., Origlia, L., \& Rich, R. M. 2011, MNRAS, 414, 2690

Valenti, E., Zoccali, M., Renzini, A., et al. 2013, A\&A, 559, A98
VandenBerg, D. A., Bergbusch, P. A., Ferguson, J. W., \& Edvardsson, B. 2014, ApJ, 794, 72

VandenBerg, D. A., Brogaard, K., Leaman, R., \& Casagrande, L. 2013, ApJ, 775,134

Watkins, L. L., van der Marel, R. P., Bellini, A., \& Anderson, J. 2015, ApJ, 803, 29 Zinn, R. 1980, ApJ, 241, 602

Zinn, R., \& West, M. J. 1984, ApJS, 55, 45

Zoccali, M., Renzini, A., Ortolani, S., et al. 2003, A\&A, 399, 931

Zoccali, M., Renzini, A., Ortolani, S., Bica, E., \& Barbuy, B. 2001, AJ, 121, 2638

Zoccali, M., \& Valenti, E. 2016, PASA, 33, e025 